UNIVERSTTÉ DU QUÉBEC À CHICOUTIMI

MÉMOIRE PRÉSENTÉ À

L'UNIVERSITÉ LAVAL

COMME EXIGENCE PARTIELLE

DE LA MAÎTRISE EN MÉDECINE EXPÉRIMENTALE

OFFERTE À

L'UNIVERSITÉ DU QUÉBEC À CHICOUTIMI

EN VERTU D'UN PROTOCOLE D'ENTENTE

AVEC L'UNIVERSITÉ LAVAL

PAR

THÉRÈSE LANDRY

TRISOMIE 21

ÉTUDE DE CONSANGUINITÉ ET D'APPARENTEMENT

AU SAGUENAY LAC ST-JEAN

OCTOBRE 1997 


\section{Bibliothèque}

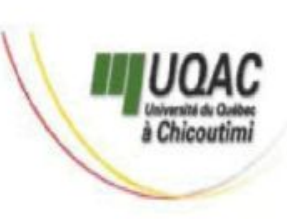

\section{Mise en garde/Advice}

Afin de rendre accessible au plus Motivated by a desire to make the grand nombre le résultat des results of its graduate students' travaux de recherche menés par ses research accessible to all, and in étudiants gradués et dans l'esprit des accordance with the rules règles qui régissent le dépôt et la governing the acceptation and diffusion des mémoires et thèses diffusion of dissertations and produits dans cette Institution, theses in this Institution, the I'Université du Québec à Université du Québec à Chicoutimi (UQAC) est fière de Chicoutimi (UQAC) is proud to rendre accessible une version make a complete version of this complète et gratuite de cette œuvre. work available at no cost to the reader.

L'auteur conserve néanmoins la The author retains ownership of the propriété du droit d'auteur qui copyright of this dissertation or protège ce mémoire ou cette thèse. thesis. Neither the dissertation or Ni le mémoire ou la thèse ni des thesis, nor substantial extracts from extraits substantiels de ceux-ci ne it, may be printed or otherwise peuvent être imprimés ou autrement reproduced without the author's reproduits sans son autorisation. permission. 


\section{RÉSUMÉ}

Une étude épidémiologique a été effectuée dans la région du Saguenay Lac St-Jean à partir de 144 caryotypes d'enfants atteints de trisomie 21 répertoriés au Centre hospitalier de Chicoutimi (l'unique établissement dans la région 02 spécialisé dans l'interprétation du nombre et de la structure des chromosomes).

Une étude génétique a été réalisée avec 67 enfants atteints de la trisomie 21 dont chacun a été apparié avec trois groupes témoins. La reconstruction généalogique a permis de constater que le coefficient moyen d'apparentement du groupe trisomique 21 est légèrement augmenté par rapport à celui du groupe témoin. Cette augmentation n'est pas statistiquement significative $(P>0,05)$. Le coefficient moyen de consanguinité est plus élevé dans le groupe trisomique 21 que dans le groupe contrôle. Cependant, cette différence n'est pas statistiquement significative $(P>0,05)$.

La prévalence des enfants atteints de trisomie 21 qui sont nés de 1975 à 1990 est de $1 / 1219,5$. Cette prévalence n'est qu'une sous-estimation compte tenu de certains facteurs qui influencent l'exactitude des données.

Cette recherche expérimentale a également permis de constater :

- Qu'il n'y a pas de localité spécifique dans la région 02 indiquant une plus grande concentration d'enfants atteints de trisomie 21 .

- Que la moyenne de l'âge maternel des proposants se situe à 35,4 ans par rapport à 27,9 ans pour les trois groupes témoins, de même que la moyenne de l'âge paternel est à 37,7 ans en comparaison avec 31,1 ans pour les trois groupes témoins.

- Qu'il n'y a pas plus d'ancêtres communs chez les enfants atteints que dans les groupes témoins.

- Que chez les proposants le sexe masculin domine par rapport au sexe féminin. Ce résultat est statistiquement significatif $(P<0,05)$. 


\section{REMERCIEMENTS}

J'exprime tout particulièrement ma gratitude à mon directeur de mémoire, madame Evelyne Heyer, ainsi qu'au responsable du programme de deuxième cycle en médecine expérimentale, monsieur Marc Tremblay, pour leur grande compréhension à mon égard et la vulgarisation de leurs connaissances.

Je remercie monsieur Marc De Braekeleer qui a permis l'amorce de cette étude.

J'adresse un remerciement spécial à madame Andrée Cholette, médecin pathologiste du Centre hospitalier de Chicoutimi, pour sa grande disponibilité lors de la collecte des données.

Je remercie l'équipe de travail d'IREP pour l'accès à son fichier informatisé de même que l'accueil chaleureux manifesté tout au long du cheminement de ma recherche. 


\section{TABLE DES MATIÈRES}

RÉSUMÉ $\ldots \ldots \ldots \ldots \ldots \ldots \ldots \ldots \ldots \ldots \ldots \ldots \ldots \ldots \ldots \ldots \ldots$

REMERCIEMENTS $\ldots \ldots \ldots \ldots \ldots \ldots \ldots \ldots \ldots \ldots$ iii

TABLE DES MATIÈRES $\ldots \ldots \ldots \ldots \ldots \ldots \ldots \ldots$ iv

LISTE DES TABLEAUX $\ldots \ldots \ldots \ldots \ldots \ldots \ldots \ldots \ldots \ldots$ vi

LISTE DES FIGURES $\ldots \ldots \ldots \ldots \ldots \ldots \ldots \ldots \ldots$ vii

LISTE DES ANNEXES $\ldots \ldots \ldots \ldots \ldots \ldots \ldots \ldots \ldots \ldots \ldots \ldots \ldots$ viii

INTRODUCTION $\ldots \ldots \ldots \ldots \ldots \ldots \ldots \ldots \ldots \ldots \ldots \ldots$

CHAPITRE 1 - PROBLÉMATIQUE $\ldots \ldots \ldots \ldots \ldots \ldots \ldots \ldots$

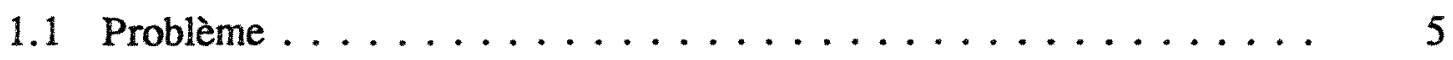

1.2 Objectifs de la recherche $\ldots \ldots \ldots \ldots \ldots \ldots \ldots \ldots$

1.3 Délimitations de la recherche $\ldots \ldots \ldots \ldots \ldots \ldots \ldots \ldots$

CHAPITRE 2 - LA TRISOMIE $21 \ldots \ldots \ldots \ldots \ldots \ldots \ldots$

2.1 Phénotype et pronostic de la trisomie $21 \ldots \ldots \ldots \ldots \ldots \ldots . \ldots \ldots$

2.2 Diagnostic de la trisomie $21:$ le caryotype . . . . . . . 20

2.3 Facteurs étiologiques connus de la trisomie 21 et leur fréquence... 24

2.4 Effet de l'âge maternel . . . . . . . . . . . . . 33

2.5 Gènes localisés sur le chromosome 21 associés au phénotype .... . 35

2.6 Diagnostic prénatal . . . . . . . . . . . . . 38

CHAPITRE 3 - MÉTHODOLOGIE $\ldots \ldots \ldots \ldots \ldots \ldots \ldots$

3.1 Collecte des données $\ldots \ldots \ldots \ldots \ldots \ldots \ldots \ldots \ldots$

3.2 Données démographiques . . . . . . . . . . . . . 47

3.3 Construction des groupes témoins . . . . . . . . . . . 49

3.4 Étude généalogique des proposants et des groupes témoins . . . . . . 50 
3.5 Calcul de la consanguinité $(\mathrm{F})$ et de l'apparentement (PHI) des proposants et des groupes témoins ............... 51

3.6 Considérations éthiques ......................... 52

CHAPITRE $4-$ RÉSULTATS $\ldots \ldots \ldots \ldots \ldots \ldots \ldots \ldots \ldots$

4.1 Configurations chromosomiques ................. 54

4.2 Calcul de la consanguinité et de l'apparentement des proposants et des groupes témoins $\ldots \ldots \ldots \ldots \ldots \ldots \ldots \ldots$

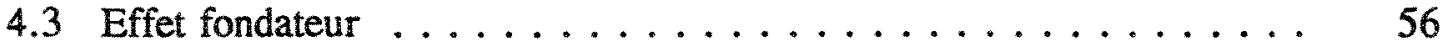

4.4 Calcul de la prévalence ..................... . . 57

4.5 Fréquence de la trisomie 21 dans les localités du Saguenay Lac St-Jean ............................. 58

4.6 Moyenne d'âge maternel et paternel des proposants et des groupes témoins ......................... 59

4.7 Fréquence des enfants atteints de trisomie 21 selon le mois de

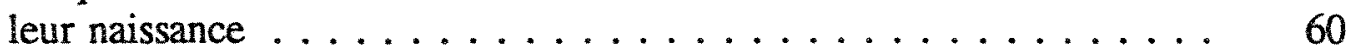

4.8 Rapport de masculinité des proposants ............... 61

CHAPITRE 5 - DISCUSSION ET RECOMMANDATIONS . . . . . . . 62

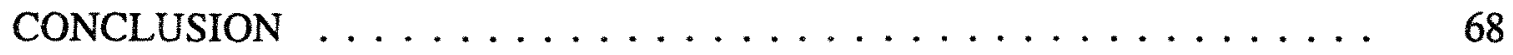

BIBLIOGRAPHIE ............................ 70

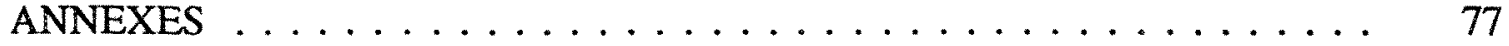




\section{LISTE DES TABLEAUX}

Tableau 1

Fréquence des configurations chromosomiques . . . . . . . . . . . 54

\section{Tableau 2}

Coefficient moyen de consanguinité $(\mathrm{F})$, coefficient de parenté $(\mathrm{PHI})$ et

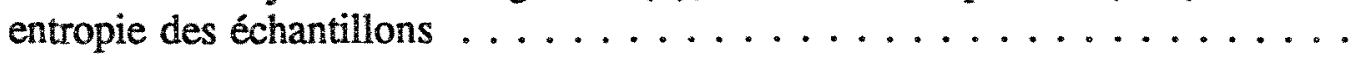

\section{Tableau 3}

Occurrence des fondateurs $\ldots \ldots \ldots \ldots \ldots \ldots \ldots$

\section{Tableau 4}

Calcul de la prévalence des trisomiques 21 de 1975 à 1990 . . . . . . . . .

\section{Tableau 5}

Fréquence de la trisomie 21 par localités au Saguenay Lac St-Jean (1975 à 1990)

\section{Tableau 6}

Moyenne et variance de l'âge maternel et paternel des proposants et des trois groupes témoins $\ldots \ldots \ldots \ldots \ldots \ldots$

\section{Tableau 7}

Fréquence des enfants trisomiques selon le mois de naissance $\ldots . . . \ldots$

\section{Tableau 8}

Fréquence des proposants selon le sexe $\ldots \ldots \ldots \ldots \ldots$ 


\section{LISTE DES FIGURES}

\section{Figure 1}

Caryotype normal .......................... 21

Figure 2

Caryotype d'un enfant atteint de trisomie $21 \ldots \ldots \ldots$

Figure 3

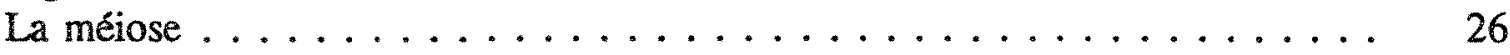

\section{Figure 4}

Translocation $21 / 21 \ldots \ldots \ldots \ldots \ldots \ldots$

\section{Figure 5}

Translocation $14 / 21 \ldots \ldots \ldots \ldots \ldots \ldots$

\section{Figure 6}

Translocation robertsonienne équilibrée $\ldots \ldots \ldots \ldots \ldots$

\section{Figure 7}

Porteur d'une translocation robertsonienne $\ldots \ldots \ldots \ldots \ldots$

\section{Figure 8}

Gènes associés au phénotype de la trisomie $21 \ldots \ldots . \ldots \ldots$ 


\section{LISTE DES ANNEXES}

\section{Annexe 1}

Représentation des lieux géographiques de la population cible :

la région du Saguenay et ses sous-régions $\ldots \ldots \ldots \ldots \ldots$

\section{Annexe 2}

Technique de prélèvement du caryotype . . . . . . . . . . . . 79

\section{Annexe 3}

Risques associés à l'âge maternel, de même que la fréquence (\%) du syndrome de Down par rapport à d'autres anomalies ................

\section{Annexe 4}

Instrument de mesure pour la collecte des caryotypes $\ldots \ldots \ldots \ldots$

\section{Annexe 5}

Collecte des données effectuée par les médecins - Identification du proposant .

\section{Annexe 6}

Méthodes de diagnostic prénatal $\ldots \ldots \ldots \ldots \ldots$

\section{Annexe 7}

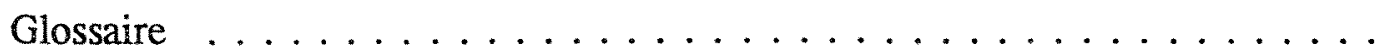


NTRODUCTION 
Certains auteurs qualifient la région du Saguenay Lac St-Jean comme une terre d'observation particulièrement utile pour les chercheurs généticiens et épidémiologistes. Cette région québécoise qui se caractérise par une très grande fréquence de porteurs de certains gènes défectueux offre à ces chercheurs une panoplie d'outils qui permettent d'informer la population, si elle le souhaite, sur les probabilités de transmettre une maladie héréditaire à leur descendance.

Parallèlement, ces calculs de probabilité de transmission de gène délétère s'avèrent un outil indispensable lorsqu'il s'agit de vérifier ou plutôt de répondre à un questionnement : à savoir si une aberration chromosomique (ou une autre pathologie génétique) présente dans cette même population s'explique en partie par les conditions démographiques ancestrales.

Les calculs de consanguinité et d'apparentement permettent de savoir si un lien peut être établi entre des personnes atteintes d'une pathologie et leur ascendance.

La présente recherche étudie particulièrement cet aspect pour une pathologie spécifique : la trisomie 21 . Cette anomalie chromosomique présente une gravité de la symptomatologie, dont les impacts sont sérieux pour chacun des individus atteints et pour une population entière. 
Le premier chapitre élabore la problématique de la trisomie 21 , précise les objectifs de même que les délimitations de la recherche.

Le deuxième chapitre présente le profil d'un enfant atteint de la trisomie 21 ainsi que la recension des écrits se rapportant à la manifestation, au pronostic, aux facteurs étiologiques connus de cette pathologie et au diagnostic prénatal.

Les trois chapitres suivants sont consacrés à la démarche méthodologique, aux résultats, à la discussion et aux recommandations. 
CHAPITRE 1

\section{LA PROBLÉMATIQUE}




\subsection{Problème}

Dans l'histoire de l'évolution de la génétique, dès 1930 l'apport des disciplines comme la biologie moléculaire et la cytogénétique, a permis à l'homme d'établir sa propre carte génétique (son caryotype). Le développement de la cytogénétique a permis très rapidement d'associer un certain nombre d'états pathologiques à une modification du caryotype (représentation graphique d'arrangements chromosomiques).

Tout réarrangement, anomalie ou aberration chromosomiques représentent une panoplie d'événements cliniques sérieux, autant pour un enfant en stage de développement qu'un enfant naissant.

Pour un enfant à l'état embryonnaire, plusieurs auteurs soulignent la gravité de telles anomalies sur la mortalité intra-utérine : "Les anomalies chromosomiques constituent la cause principale des échecs de fécondation, tant in vivo qu'in vitro " (Pellestor F. 1990); « De nombreuses études caryotypiques effectuées sur les avortats, ont montré l'importance des trisomies qui représentent près de $60 \%$ des avortats de moins de 12 semaines " (Serre J.-L. et coll. 1983).

Un même auteur cite: «Ces aberrations chromosomiques constituent de véritables mutations de l'ensemble du génome et non pas d'un ou de plusieurs gènes en particulier " 
(Pellestor F. 1990). Du point de vue démographique, l'augmentation du taux de mortalité intra-utérin influence le taux de fécondité d'une population.

Cependant, parmi les anomalies chromosomiques autant structurales que numérales, c'est la découverte de la trisomie 21 par Lejeune et coll. qui a ouvert l'ère de la cytogénétique humaine en 1959. L'identification du chromosome 21 a été fondée sur la clinique; c'est par définition le chromosome dont la présence à l'état trisomique est responsable du syndrome clinique, d'abord décrit sous le nom de mongolisme (De Grouchy J., Turleau C. 1977).

La trisomie 21 représente l'aberration chromosomique la plus fréquente et de plus, elle est la principale cause d'arriération mentale (Fattorusso V., Ritter O. 1986). Cette affection congénitale modifie non seulement le génotype et le phénotype de tout individu atteint, mais influence par surcroît la vie et la qualité de vie de celui-ci.

Outre la gravité de la symptomatologie, les enfants atteints de trisomie 21 doivent subir le phénomène de l'intolérance sociale. En effet, la tolérance sociale vis-à-vis les trisomiques a beaucoup baissé depuis une dizaine d'années. Une enquête, réalisée dans 26 maternités des Bouches-du-Rhône sur une population de 280 trisomiques 21 nés en huit ans, a montré que la proportion d'enfants abandonnés à la naissance est passée de $2 \%$ en $1984,13 \%$ pour la période $1986-1988$ et $20 \%$ pour les années 1989-1990. En 
tête de ce triste palmarès, les parents de nationalité française et de niveau social élevé, surtout lorsqu'il s'agit d'un premier-né (Petitnicolas C. 1994).

L'impact biopsychosocial d'un enfant atteint et la dimension psychoaffective des parents représentent des motifs intéressants d'effectuer une étude sur la trisomie 21 .

Plusieurs auteurs ont émis des hypothèses concernant les facteurs étiologiques de la trisomie 21 : l'âge maternel avancé (Serre J.-L. et coll. 1983) de même que l'existence d'un facteur génétique pouvant influencer cette pathologie (Alfi O. S. et coll. 1980; Roberts D. F. et coll. 1991). Ces hypothèses formulées suggèrent une panoplie d'interrogations d'où l'intérêt d'obtenir des réponses par une étude épidémiologique et génétique de cette pathologie dans une population cible qui présente un taux plus élevé de certaines maladies héréditaires que d'autres régions du Québec.

Cette région cible, le Saguenay Lac St-Jean, n'offrait pas de critères sélectifs prédominants de cette pathologie mais elle représentait un choix judicieux, compte tenu de l'absence de recherche antérieure sur le sujet dans la région. Cette région offrait également l'opportunité d'établir avec certitude le diagnostic médical de cette pathologie. La figure représentant les lieux géographiques de la population cible est visualisée dans l'annexe 1. 
Cette anomalie chromosomique quantitative ou numérale décrite par John Langdon Down en 1866 et désignée syndrome de Down, résulte d'un chromosome 21 surnuméraire et présente un caryotype de 47 chromosomes.

L'incidence de cette pathologie demeure stable depuis plusieurs années. En 1969, l'incidence du syndrome de Down est de 1,45 sur mille, soit 1 pour 700 naissances (Lilienfeld A.M., Benesch C.H. 1969). En 1981, l'incidence du syndrome de Down est aussi de 1 cas par 700 naissances (Solomon E. P., Davis P. W. 1981). En 1994, un auteur décrit le même taux d'incidence (Patterson D. 1994).

Cette fréquence parait constante dans tous les pays développés; un enfant parmi 700 à 1000 naissances est atteint du syndrome de Down (Baird P., Sadovnick A. 1988; Thompson I. M., Thompson D. D. 1988; Wald N.J. et coll. 1988).

\subsection{Objectifs de la recherche}

L'objectif primordial de cette recherche consistait à savoir si un lien peut être établi entre les enfants diagnostiqués trisomiques 21 et leur ascendance (5 générations). Le calcul de la consanguinité et d'apparentement de ces enfants atteints par rapport au 
groupe contrôle permettait d'interpréter certaines hypothèses concernant les facteurs étiologiques de la trisomie 21 dans une population cible, le Saguenay Lac St-Jean.

\section{Objectifs secondaires}

Conjointement à la possibilité de vérifier le facteur de risque lié à l'âge maternel d'enfants trisomiques 21 , il m'apparaissait également pertinent de connaître l'existence d'une concentration particulière de ces mêmes enfants dans la région du Saguenay Lac St-Jean, d'où l'opportunité d'observer un facteur de risque environnemental.

Parmi les objectifs secondaires de ma recherche, je m'étais fixée un objectif particulier; celui d'appuyer les observations pertinentes du docteur Jean Larochelle (pédiatre) qui avait noté tout au long de sa carrière professionnelle que les naissances d'enfants trisomiques 21 semblaient privilégier une saison particulière durant l'année (le printemps).

D'autres objectifs s'ajoutent à ma recherche comme celui de calculer la prévalence de la trisomie 21 ainsi que d'établir le rapport de masculinité des enfants atteints répertoriés pour cette étude. Outre ces considérations, l'étude de l'aspect global de cette pathologie m'apportera des dimensions académiques supplémentaires qui me permettront d'élargir ma compétence en conseil génétique. 


\subsection{Délimitations de la recherche}

La recherche s'est effectuée dans la région du Saguenay Lac St-Jean. Le Centre hospitalier de Chicoutimi (département de pathologie) est le seul établissement de santé dans cette région capable d'effectuer un caryotype et d'interpréter fidèlement le résultat. 


\section{CHAPITRE 2}

LA TRISOMIE 21 
La trisomie 21 est une aberration chromosomique génétique qui représente pour un enfant atteint un ensemble d'anomalies physiques et mentales très graves. La triplication de la plus petite région du chromosome 21 est nécessaire pour provoquer le phénotype de cette pathologie. La présence du 21 en trois exemplaires n'est pas indispensable car une seule zone étroite du bras long est responsable du syndrome de Down (Huret J.L. et coll. 1990).

\subsection{Phénotype et pronostic de la trisomie 21}

\section{Dysmorphie cranio-faciale}

Le crâne est petit et rond. L'occiput est plat. La nuque est courte, plate et large avec un excès de peau; il s'agit d'un excellent signe qui permet souvent de faire le diagnostic en voyant l'enfant de dos ou de trois-quarts arrière. Le visage est rond avec un profil plat. Le front est normalement bombé (De Grouchy J., Turleau C. 1977).

Les fentes palpébrales sont très nettement obliques en haut et en dehors. Leur angle interne est masqué par un épicanthus (un repli de la peau qui recouvre la commissure interne de l'oeil) donnant souvent une fausse impression d'hypertélorisme (élargissement de la petite aile du sphénoïde, donc de l'espace interorbitaire et de la 
racine du nez et par un écartement excessif des yeux). Les cils sont rares et courts. Une blépharite (inflammation des paupières) est fréquente.

Les iris montrent, principalement lorsqu'ils sont bleus, un signe presque pathognomonique (signe appartenant à une pathologie spécifique) : l'existence de taches de Brushfield. Il s'agit de petites taches blanchâtres rondes, un peu irrégulières et formant une couronne située à la jonction du tiers moyen et du tiers externe de l'iris. Il existe fréquemment un strabisme. La plupart des enfants trisomiques 21 ont des anomalies du cristallin, qui augmentent les risques de cataracte et de troubles visuels (Patterson D. 1994).

Les trisomiques 21 ont une sensibilité pupillaire marquée à l'atropine, à la pilocarpine, à l'éphédrine qui semble correspondre à un déficit cholinergique (De Grouchy J., Turleau C. 1977). La racine du nez est plate du fait de l'hypoplasie des os propres. Le nez est court et les orifices narinaires sont visibles de face. La bouche est petite. Les lèvres sont épaisses et peuvent être crevassées. La langue est souvent grosse et protuse. Il peut exister une glossite exfoliatrice (desquamation de la première couche superficielle de la langue).

Les oreilles sont petites et rondes. Le bord supérieur de l'hélix forme un repli horizontal. La hauteur de l'oreille est réduite. Le lobule est petit et adhérent. Un signe 
très évocateur est le développement anormal de la racine de l'hélix qui traverse entièrement la conque. Le conduit auditif est petit et l'on observe fréquemment un crux cymbae.

\section{Thorax et abdomen}

Du fait de l'hypotonie, l'abdomen est distendu avec souvent un diastasis des droits et une hernie ombilicale. Le bassin est plutôt petit et les angles acétabulaires (cavité iliaque supportant la tête du fémur) et iliaques sont toujours diminués sur les clichés radiologiques (De Grouchy J., Turleau C. 1977).

\section{Membres}

La main est large et trapue. Les doigts sont courts, surtout le cinquième et le pouce. Il existe une brachymésophalangie (brièveté de la $2^{\mathrm{e}}$ phalange) et une clinodactylie du $5^{\mathrm{e}}$ (déviation des doigts vers la face dorsale, la face palmaire ou plantaire consécutive à des rétractions cicatricielles ou ligamenteuses) qui sont très caractéristiques avec parfois un seul pli de flexion. Le pied est large, petit et plat avec des orteils courts. Les deux premiers orteils sont trop espacés. 


\section{Organes génitaux}

Les organes génitaux sont normaux.

\section{Malformations cardiaques, digestives et osseuses}

Les malformations viscérales sont fréquentes; les plus importantes sont les malformations cardiaques qui frappent $40 \%$ des trisomiques (Patterson D. 1994). Ce sont par ordre de fréquence : le canal atrioventriculaire,les communications interventriculaires et interauriculaires, la persistance du canal artériel (Schneider D. et coll. 1989). Les malformations digestives comprennent essentiellement la sténose duodénale. Un tiers des sténoses duodénales surviennent chez des trisomiques 21 . Un pancréas annulaire, une atrésie anale, un mégacôlon et un prolapsus rectal sont également observés.

Les anomalies osseuses sont évocatrices. Celles du bassin, déjà signalées, sont les plus fréquentes. On peut retenir également la brièveté de la deuxième phalange du cinquième doigt, l'existence de deux points d'ossification du manubrium sternal (partie supérieure du sternum), l'absence de la douzième côte, la microcéphalie inconstante, l'absence de sinus frontaux, la persistance de la suture métopique (suture médiane reliant les deux 
bosses frontales). L'âge osseux est le plus souvent un peu retardé. Aucune anomalie constante du système nerveux central n'a pu être mise en évidence (De Grouchy J., Turleau C. 1977).

\section{Retard mental}

Le déficit mental de la trisomie 21 peut varier dans des limites assez larges selon les individus. Il varie également en fonction de l'âge. En moyenne, le QI se situe à 50 à l'âge de 5 ans. Il décroît ensuite de façon progressive jusqu'à une valeur moyenne de 38 vers 15 ans. Avant l'âge de 5 ans le QI est plus difficile à évaluer. Il décrô̂t jusqu'à 50 entre 2 et 3 ans, puis croît jusqu'à 58 entre 3 et 4 ans et décroît à nouveau (Prieur M. 1968).

De part et d'autre de cette évaluation moyenne, on peut voir des malades avec des QI relativement élevés de l'ordre de 70-80, d'autres condamnés à une vie végétative. Un auteur expérimente si cette décroissance du QI chez les trisomiques 21 peut être influencée par l'environnement (Brown Frank R. et coll. 1990).

D'une manière générale, ce sont surtout les facultés de raisonnement abstrait qui sont les plus touchées, alors que l'affectivité et la sociabilité sont relativement conservées 
du moins chez l'enfant. Chez l'adulte, l'acquisition d'un certain nombre d'automatismes peut masquer l'importance du retard mental. Un auteur suggère des interventions favorisant l'intégration sociale des enfants atteints de trisomie 21 (Gompertz J. 1990).

\section{Évolution de la pathologie}

Le poids et surtout la taille des sujets restent inférieurs à la normale. La taille moyenne chez le trisomique adulte est de $154 \mathrm{~cm}$ chez le garçon et de $144 \mathrm{~cm}$ chez la fille (De Grouchy J., Turleau C. 1977). La dysmorphie faciale se modifie. La rondeur du visage s'atténue, de même que l'épicanthus et l'aplatissement de la racine du nez. Une rougeur des pommettes s'installe et le visage prend un aspect vieillot et la voix est rauque.

La puberté se fait normalement dans les deux sexes. Les filles sont fécondes, bien que leur libido paraisse peu importante. On connait une vingtaine de cas de maternité chez des trisomiques 21. On observe alors, comme le veut l'hypothèse génétique, un nombre égal d'enfants disomiques et d'enfants trisomiques 21 (Rethore M.O. et coll. 1970; Scharrer S. et coll. 1975). 
Chez le garçon, aucun cas de paternité n'est prouvé. L'histologie testiculaire ne permet pas de considérer ces malades comme stériles. S'il existe une diminution de la fertilité, celle-ci serait plus vraisemblablement due à un défaut de maturation postméiotique (Kjessler B., La Chapelle A. 1971). Un auteur élabore sur la fertilité des enfants masculins atteints de trisomie 21 (Thompson I., Thompson D. 1988).

Avec l'âge on voit apparaître un vieillissement précoce, des troubles psychotiques et une cataracte sénile.

\section{Pronostic de la trisomie 21}

Le pronostic vital est conditionné par l'existence de cardiopathies, de malformations digestives, de sensibilité aux infections surtout l'hépatite B (Green J. 1988) ainsi que le pourcentage de risque accru de leucémies. Un auteur affirme que chez les personnes atteintes de trisomie 21 , le risque de leucémie est 20 à 50 fois supérieur à la normale. Cette leucémie est décrite comme étant la leucémie myélogène aiguë de type M2 (Patterson D. 1994).

Des chercheurs aux États-Unis ont effectué une étude concernant la transplantation de la moelle osseuse chez les trisomiques 21 atteints de leucémies (Arenson E.B., Forde 
D. 1989). Un auteur suggère des réflexions et des considérations éthiques concernant cette intervention chirurgicale (Churchill L.R. 1989).

Il est intéressant de comparer les statistiques sur l'espérance de vie entre 1971 et 1994.

En 1971, 25 à $30 \%$ des malades meurent au cours de la première année, $50 \%$ avant 5 ans. Pour les autres classes d'âges, la létalité demeure plus élevée que dans la population générale ( 5 à 6 fois). Il y a $8 \%$ des malades qui survivent après 40 ans et 2,6\% après 50 ans (Kjessler B., La Chapelle A. 1971).

En 1994, un auteur relate l'accroissement de l'espérance de vie au fil des années. En 1928, l'espérance de vie s'estimait à neuf ans; en 1980, cette espérance de vie est reportée à 30 ans et aujourd'hui, plus de $25 \%$ de ces malades vivent jusqu'à 50 ans (Patterson D. 1994).

Des chercheurs en Colombie-Britannique ont réalisé une étude sur l'espérance de vie des enfants trisomiques 21 (Baird P., Sadovnick A.D. 1988). Une autre étude similaire a été effectuée en Italie (Mastroiacovo P. et coll. 1990). 


\subsection{Diagnostic de la trisomie 21 : le caryotype}

La trisomie 21 est caractérisée par la présence dans les cellules d'un chromosome surnuméraire placé à côté de la paire de chromosomes homologues du même type, ce qui porte le nombre total de chromosomes à 47 au lieu de 46 (nombre total de chromosomes chez un individu normal).

Seul le caryotype diagnostique de façon précise la présence d'un chromosome surnuméraire sur la $21^{\mathrm{e}}$ paire. Sa représentation graphique d'arrangements chromosomiques (nombre, dimensions relatives et morphologie des chromosomes), confirme le phénotype observé par les médecins chez un nouveau-né.

Le caryotype confirme également la présence d'une partie si minime soit-elle de ce chromosome surnuméraire sur la $21^{\mathrm{e}}$ paire de même qu'il assure une représentation franche d'une translocation. La figure 1 présente un caryotype normal et la figure 2 présente le caryotype d'un enfant atteint de trisomie 21.

Configuration chromosomique d'un caryotype normal $46, X Y$

Configuration chromosomique d'un caryotype trisomique 21 et nomenclature $47, X Y,+21$

1 Nomenclature

1. Nombre total des chromosomes (autosomes et chromosomes sexuels).

2. Chromosomes sexuels.

3. Identification du chromosome surnuméraire. 
Figure 1

Caryotype normal
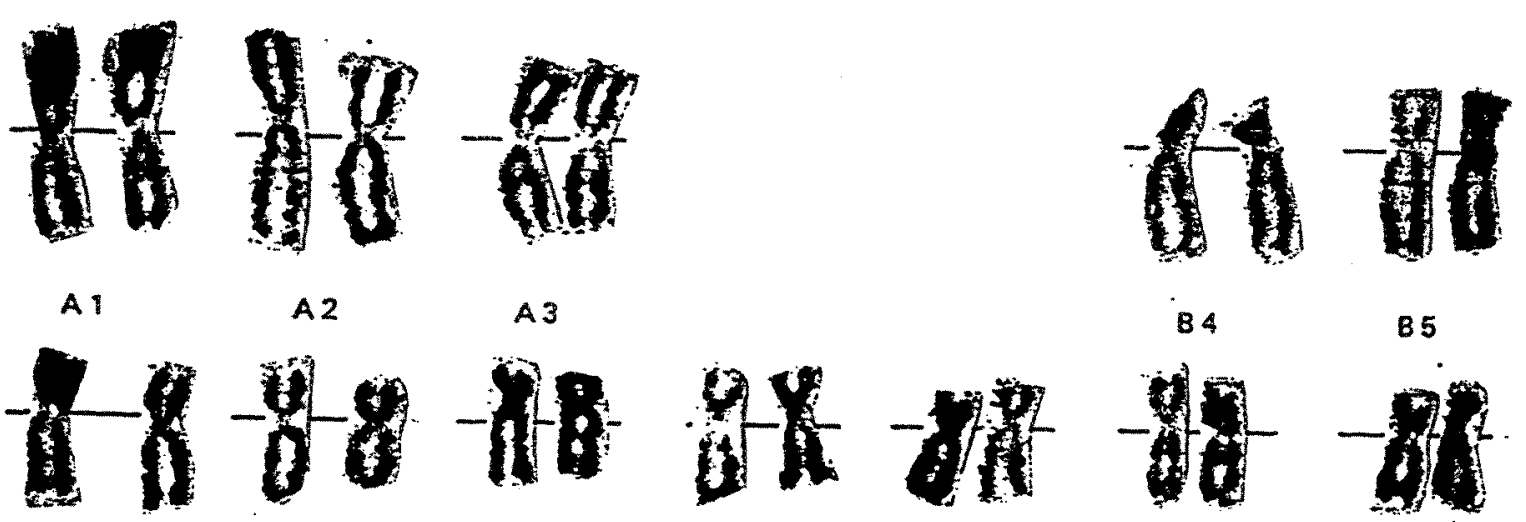

A2

A 3
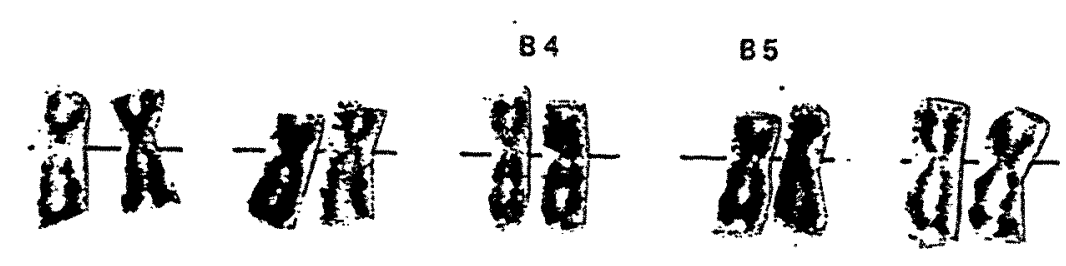

c6

C7

$C_{8}$

c9

C10

c11

Ci2

$x$
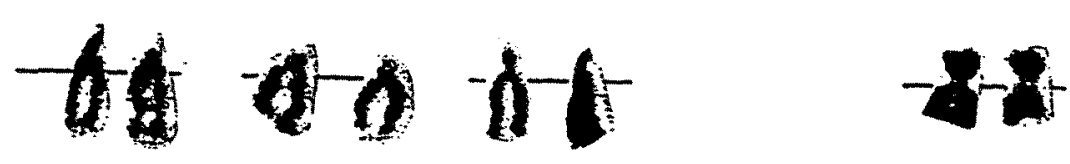

$-4$

$-1+4$

D13

D14

D15

E16

E17

E18

$-2-4-2 x-2$

$-6-4-4-4$

F19

F 20

G21

$Y$

Source: Archives médicales /Centre hospitalier Chicoutimi 
Figure 2

Caryotype d'un enfant atteint de trisomie 21
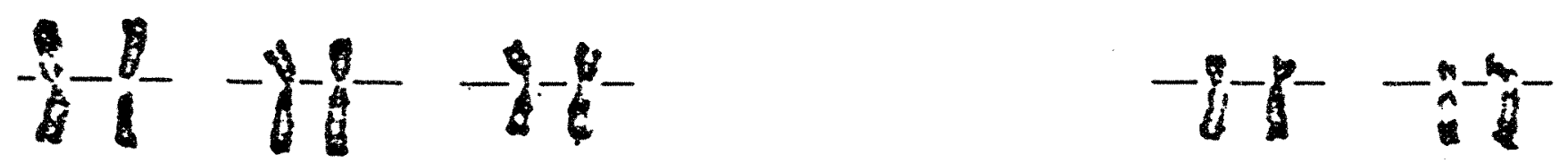

A1

A2

A3

B4

B5
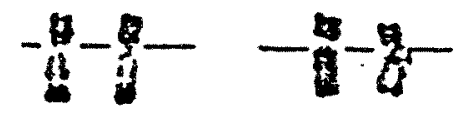<smiles>[V]</smiles><smiles>[AsH2+]</smiles><smiles>C=C[N+](C)=O</smiles><smiles>C[AsH][AsH2](C)C</smiles>

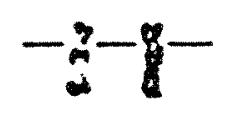<smiles>COC</smiles>

66

67

C8

C9

c10

CII

$\mathrm{Cl} 2$ $x$
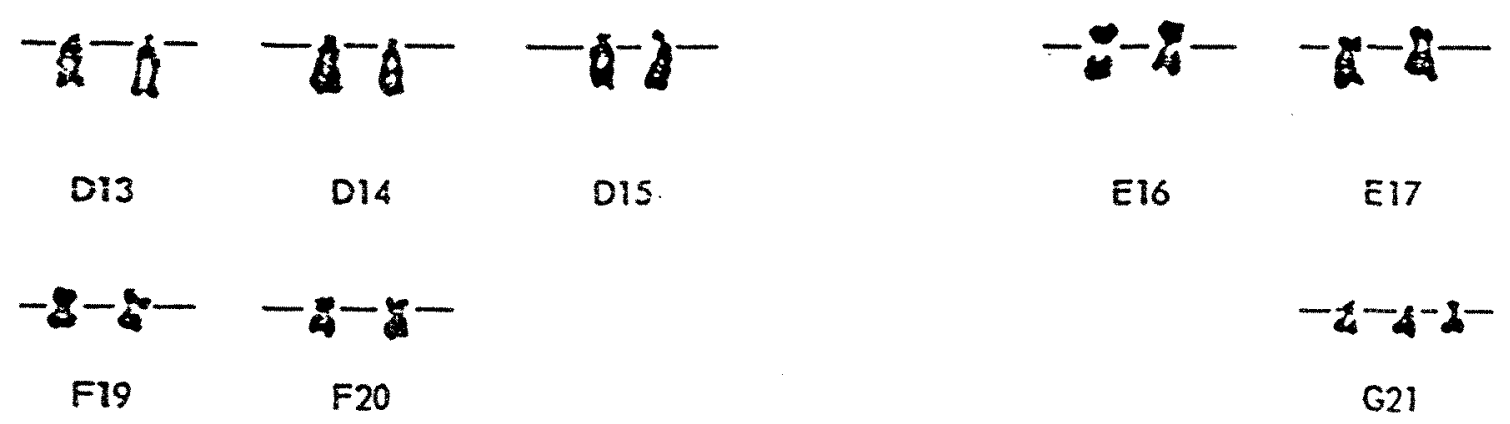

$-5-6$

D15.

E16

$E 17$

Eิa
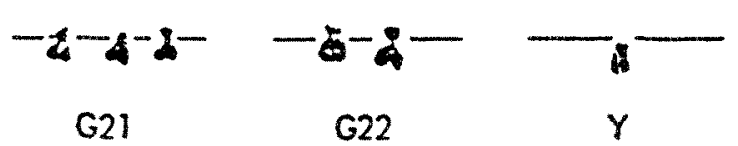

$F 20$

G21

G22

$Y$

Source: Archives médicales /Centre hospitalier Chicoutimi 
La précision d'un caryotype effectué nécessite certaines conditions particulières : une technique de prélèvement très spécialisée, des cytogénéticiennes qui provoquent en laboratoire la visualité des chromosomes, un environnement adapté qui n'interfère pas sur la structure morphologique de ces chromosomes et la présence d'un médecin pathologiste pour interpréter les résultats finaux.

\section{Technique du caryotype}

La technique de prélèvement s'effectue sur des cellules (sang, moelle osseuse, liquide amniotique, tissu provenant d'un fragment d'un oeuf fertilisé, peau, testicules) prélevées par biopsie cutanée, ponction veineuse, amniocentèse et foetoscopie.

Une seule cellule sanguine (le lymphocyte) est suffisante pour la mise en culture en laboratoire. Les mitoses sont inhibées par des agents chimiques et l'on photographie les chromosomes durant la métaphase. Après agrandissement de ces photos, les chromosomes se présentent par paires; ils sont ensuite comptés dans une cellule et l'on identifie alors les défauts visibles. Ces cellules cultivées sont extrêmement sensibles à la température du milieu (chaleur, humidité, air ambiant). 
La technique de prélèvement utilisée pour effectuer un caryotype au Centre hospitalier de Chicoutimi est présentée dans l'annexe 2.

\subsection{Facteurs étiologiques connus de la trisomie 21 et leur fréquence}

Trisomie 21 libre

Les statistiques des fréquences indiquées sont celles données par Giraud et Mattei 1975, à partir de 4760 cas de trisomie 21 .

La majorité des trisomies 21 sont des trisomies libres pouvant être considérées comme des accidents isolés. Une trisomie 21 libre est présente dans $92,5 \%$ des cas. Elle résulte d'une non-disjonction survenant lors de la première division méiotique (métaphase 1) ou de la seconde division méiotique (métaphase 2) maternelle ou paternelle (Giraud F., Mattei J. F. 1975). Des auteurs ont démontré que $40 \%$ des non-disjonctions sont d'origine paternelle (Bott C.E. et coll. 1975; Hara Y. et coll. 1975).

C'est Bridges en 1916 qui a fait la découverte de la non-disjonction. Plusieurs facteurs peuvent influencer ce phénomène : facteurs environnementaux, virus, radiations, produits chimiques ainsi que des facteurs génétiques (Alfi O.S. et coll. 1980; Roberts D. F. et coll. 1991). L'hypothèse de la génétique formulée par ces auteurs serait l'existence 
d'un gène qui influencerait la non-disjonction dans l'oeuf fertilisé chez les homozygotes. Cependant d'autres chercheurs rejettent cette hypothèse (Basaran N. et coll. 1992; Martinez-Frias M. L. 1993).

La figure 3 visualise le phénomène de non-disjonction dans l'anaphase 1 et 2 de la méiose.

Syndrome de Down par translocation

La minorité des cas de syndrome de Down sont dus à des translocations qui sont responsables de 4,8\% des cas (Giraud F., Mattei J. F. 1975). La translocation est un changement de structure chromosomique caractérisé par un changement de position de segments chromosomiques. Elle correspond à un transfert d'un segment chromosomique de sa place normale à un autre endroit du génome. Les caryotypes d'une translocation 21/21 et d'une translocation 14/21 causant le phénotype du syndrome de Down sont présentés dans les figures 4 et 5 .

Une translocation est équilibrée lorsque la reconstitution du stock génique est normale, par conséquent le phénotype est normal. Une translocation est déséquilibrée lorsqu'elle entraîne une perturbation du patrimoine génétique et du phénotype. 
Figure 3

La méiose

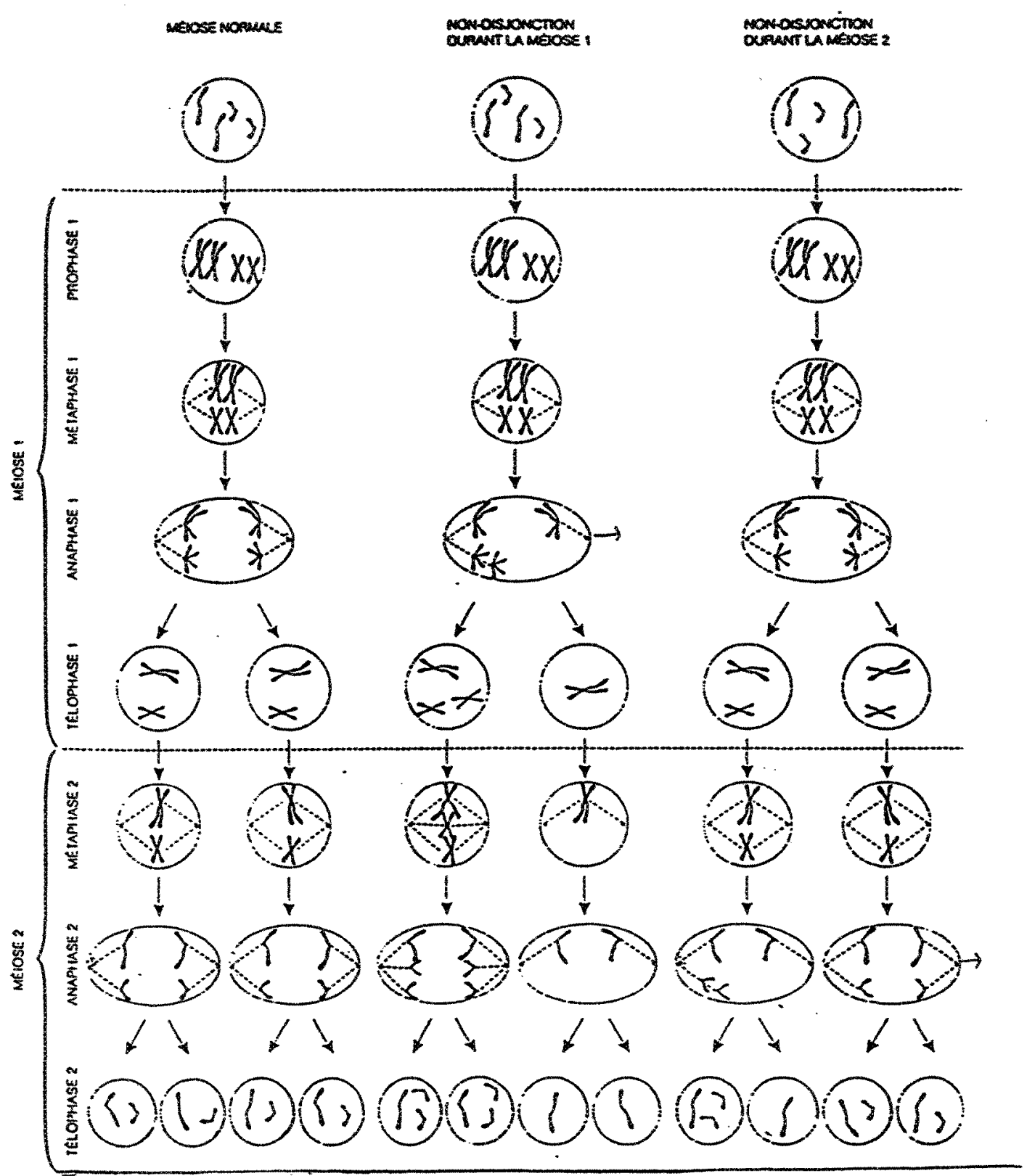

Source : Patterson David (1994). La trisomie 21. Dossier pour la science, avril, p. 5460. 
Figure 4

Translocation 21/21
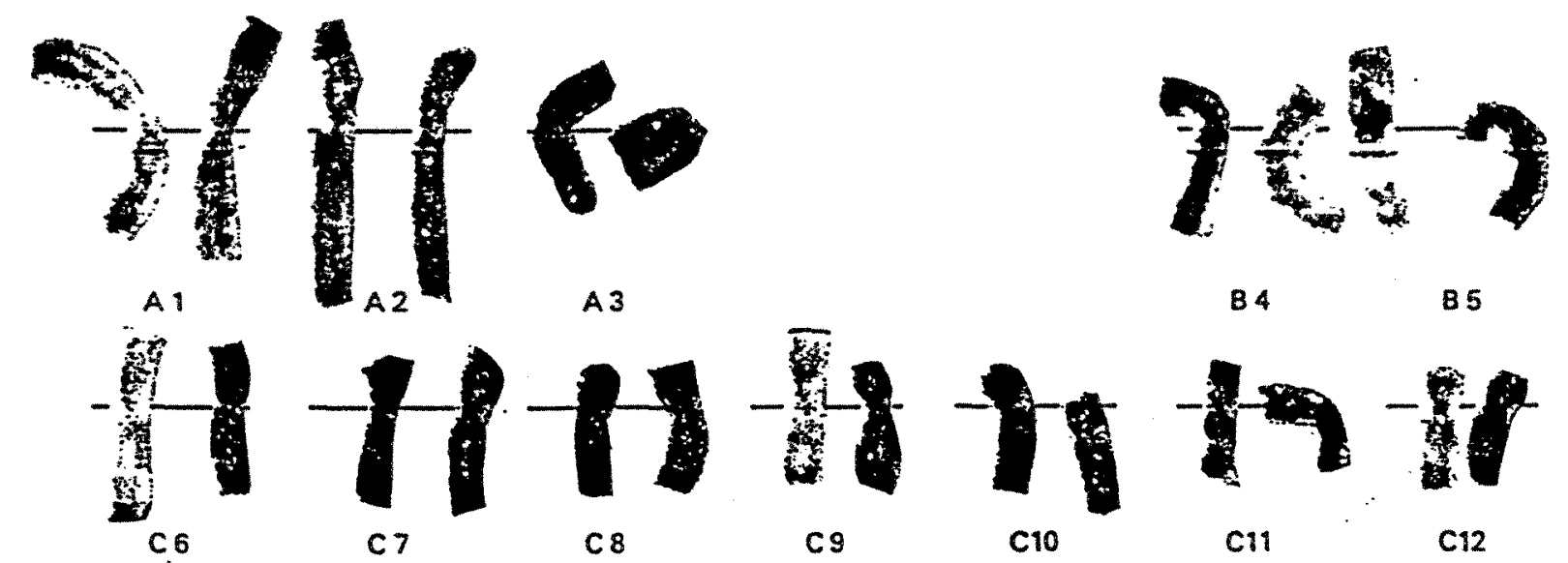

co
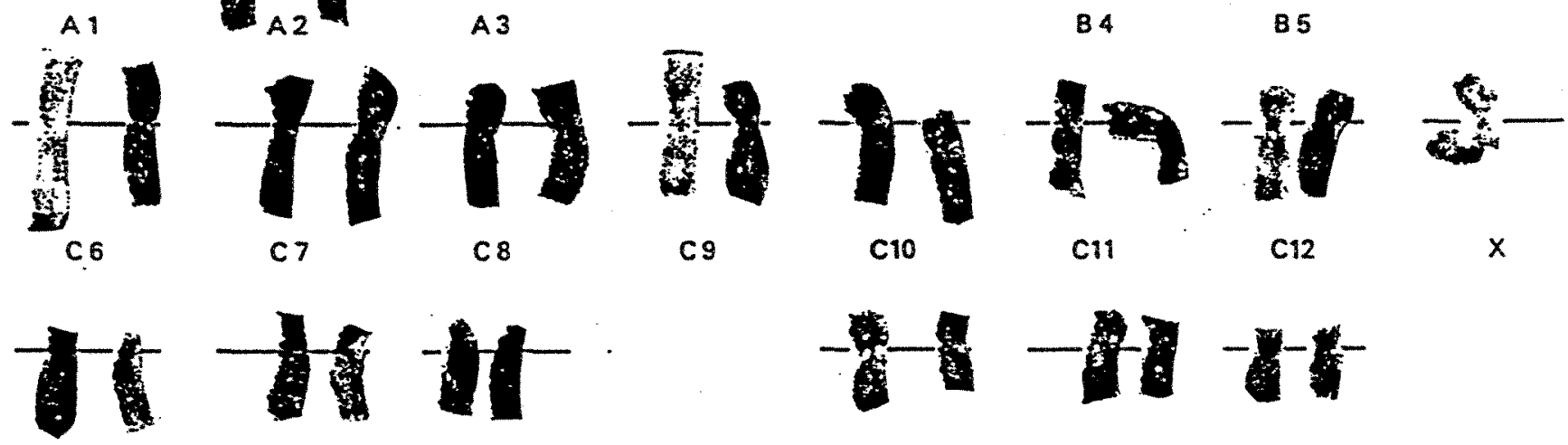

D15
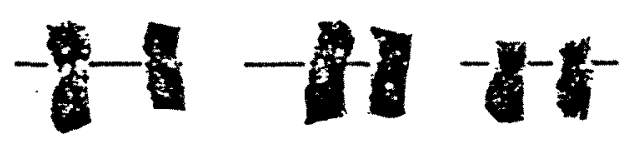

E16

E17

E18
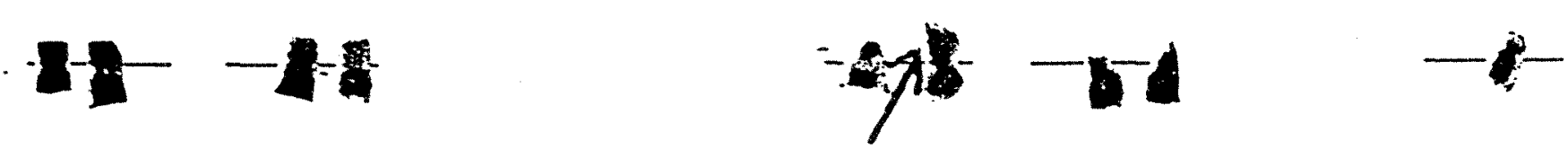

F19

F20

G21

G22

$Y$

Source : Archives médicales /Centre hospitalier Chicoutimi 
Figure 5

Translocation 14/21

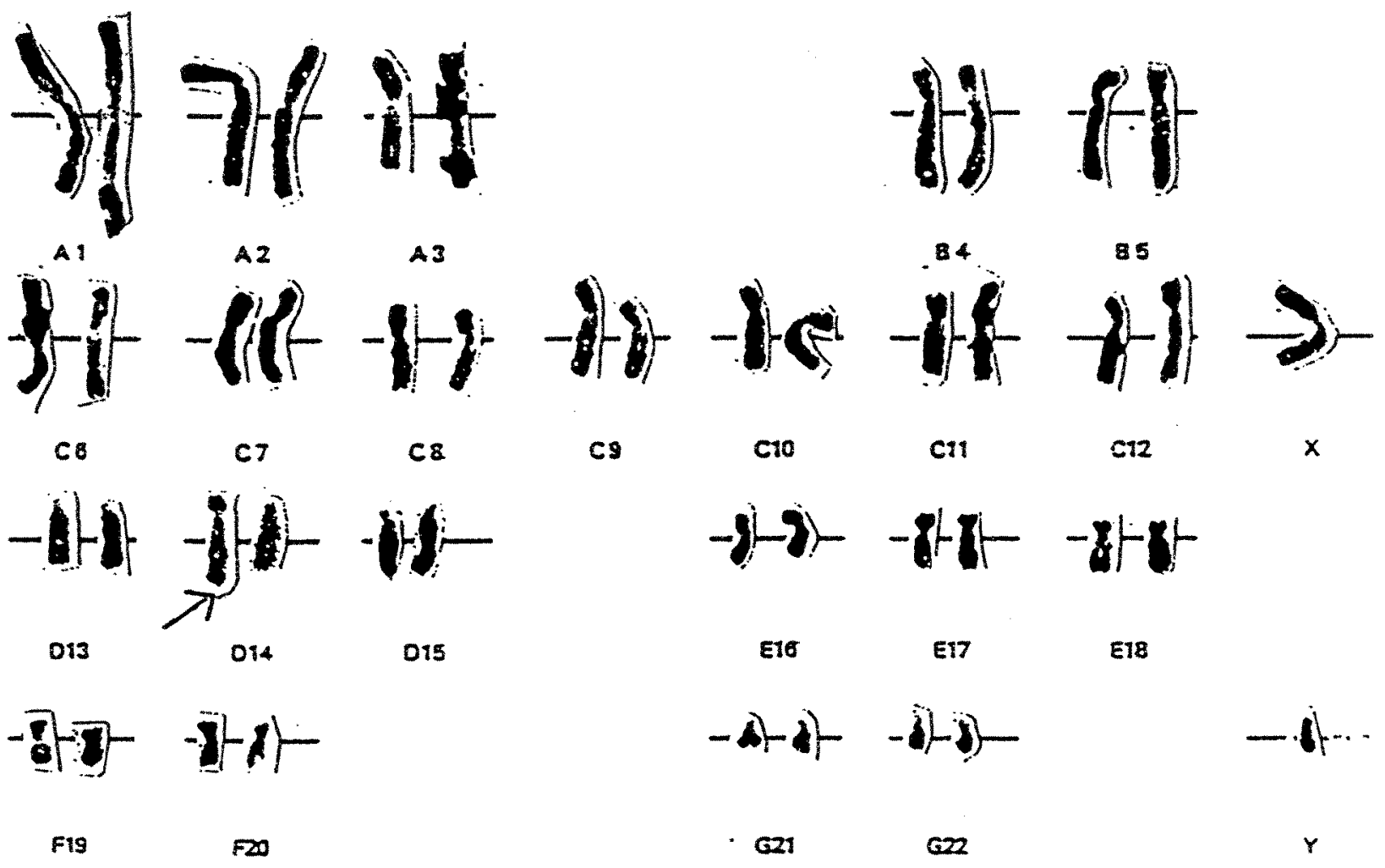

Source: Archives médicales /Centre hospitalier Chicoutimi 
Le syndrome de Down peut être acquis par un parent porteur d'une translocation équilibrée (phénotype normal, mais génotype anormal). En effet, un parent normal (en apparence) peut posséder dans son génome une translocation réciproque équilibrée (échange d'un stock normal de gènes), mais transmettre un phénotype de trisomie 21 ou un phénotype de trisomie 14 (en supposant que le chromosome 14 est impliqué dans la translocation réciproque équilibrée du porteur).

Le meilleur exemple de cette empreinte parentale est la translocation réciproque robertsonienne : fusion de deux chromosomes différents au niveau de leur centromère. L'exemple le plus fréquent chez l'homme est la fusion 14-21 représentée par un caryotype effectué aux deux parents (Dreyfus J-C. 1985).

Une translocation robertsonienne $14 \mathrm{q} 21 \mathrm{q}$ entre les bras longs (q) des chromosomes 14 et 21 donne un caryotype équilibré si elle est associée à un chromosome 14 et 21 ; en effet le stock normal de gènes d'une paire de 14 et d'une paire de 21 est reconstitué (Serre J.-L. et coll. 1983).

L'exemple d'une translocation robertsonienne équilibrée d'un porteur à phénotype normal se trouve dans la figure 6 , et la figure 7 nous montre le syndrome de Down acquis par ce porteur (père ou mère). 
Figure 6

Translocation robertsonienne équilibrée
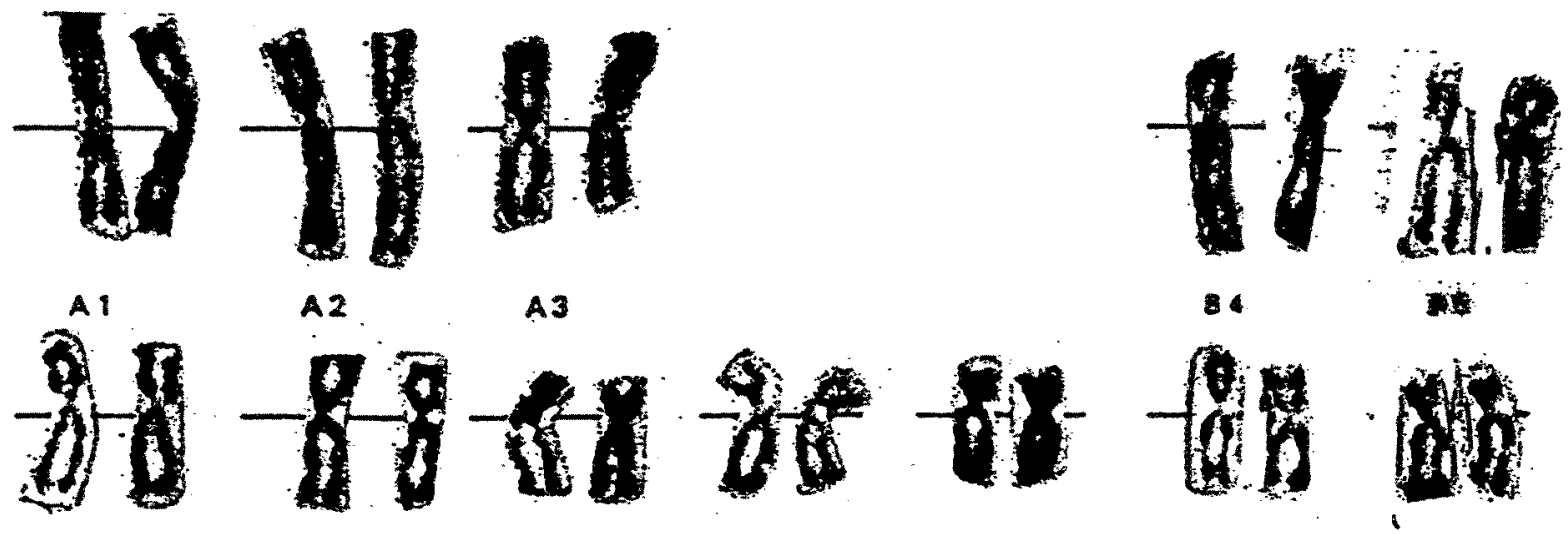

A3
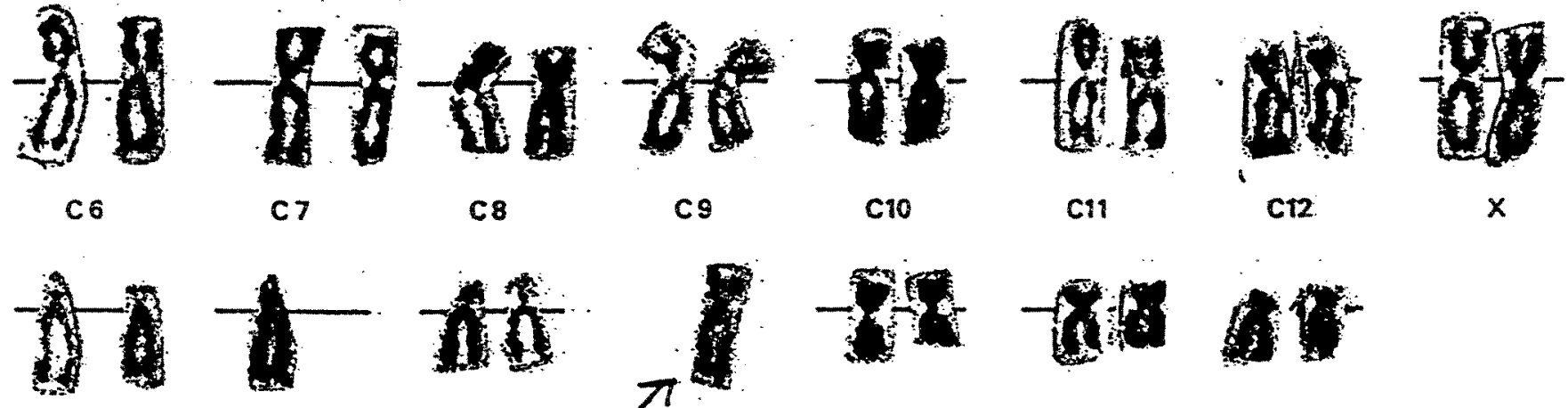

D13

014

D15.

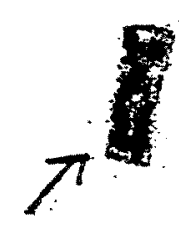

C11
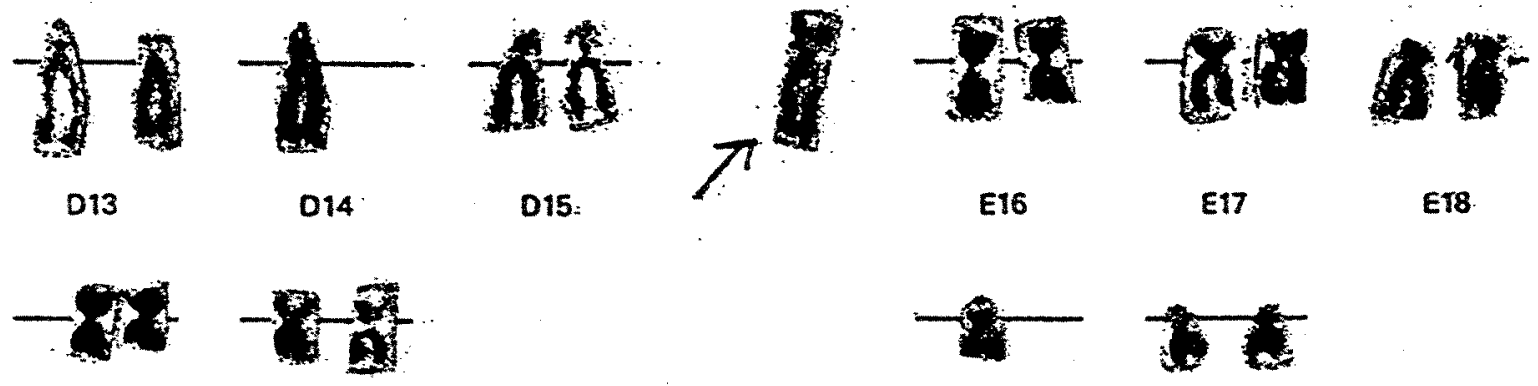

F19

$F 20$

621

G22

$Y$

Source : Archives médicales /Centre hospitalier Chicoutimi 
Figure 7

\section{Porteur d'une translocation robertsonienne}

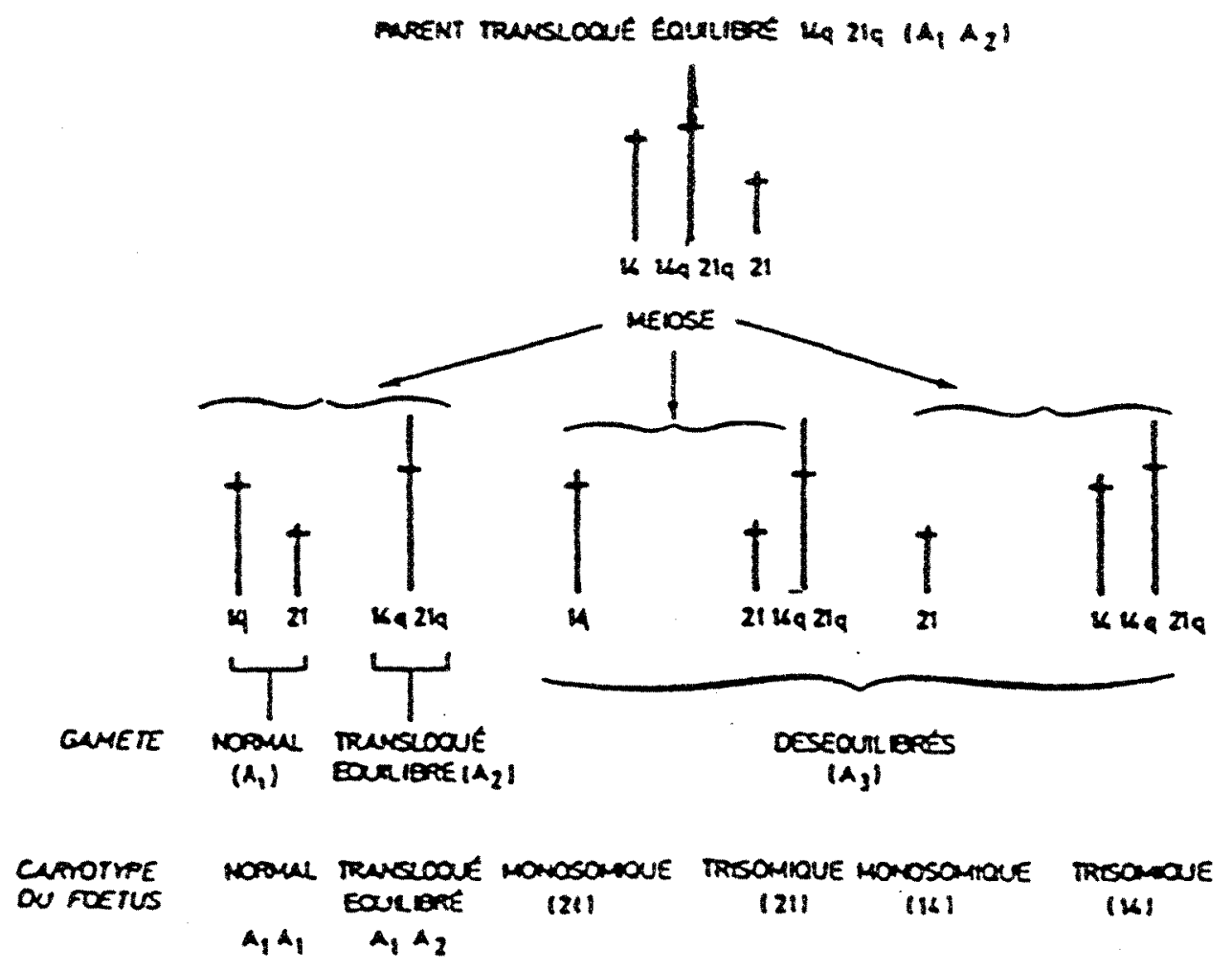

Source : Serre J.-L. Feingold J., Gallano P., Boué J., Boué A (1983). Maintien et évolution des fréquences des anomalies de structures chromosomiques : application à quelques anomalies étudiées chez l'homme. Population 2, p. 283-306. 
Formule chromosomique d'une translocation robertsonienne $45, \mathrm{XX}, \mathrm{t}(14 ; 21)(\mathrm{p} 14 ; \mathrm{q} 21)$.

Formule chromosomique d'une translocation robertsonienne $45, X Y,-14,-21,+t(14 q ; 21 q)$. Nomenciature spécifique à une translocation ${ }^{2}$

\section{Syndrome de Down par mosaïcisme}

Les mosaïques représentent $2,7 \%$ de tous les cas de syndrome de Down (Giraud F., Mattei J. F. 1975). Le mosaïcisme est une anomalie chromosomique caractérisée par la coexistence chez le même individu de lignées cellulaires qui different entre elles par leur constitution chromosomique (Fattorusso V., Ritter O. 1986).

Formule chromosomique d'une mosaïque de trisomie 21 chez une fille : $46, X X / 47, X X,+21$. Les différentes populations cellulaires sont indiquées les unes après les autres et séparées par une barre diagonale.

2 Nomenclature

1. Nombre total des chromosomes (autosomes et chromosomes sexuels).

2. Une virgule.

3. Chromosomes sexuels.

4. Le « $t$ " veut dire translocation.

5. La parenthese no. 1 identifie les chromosomes qui ont subi une cassure et la parenthèse no. 2 indique la partie conservée des deux chromosomes.

6. La dénomination " $q$ " signifie le bras long d'un chromosome par rapport à " $p$ * qui signifie le bras court d'un chromosome. 


\subsection{Effet de l'âge maternel}

Le risque de voir naître un enfant trisomique 21 augmente de façon exponentielle avec l'âge maternel. II est de 1 sur 2000 à 20 ans; il augmente peu jusqu'à 30 ans; il est de 1 sur 300 à 35 ans; de 1 sur 100 entre 40 et 45 ans; il atteint 1 sur 50 après 45 ans (Penrose L.S. 1933; Lilienfeld A.M., Benesch C.H. 1969; Mikkelsen M. 1972).

Les facteurs de risques associés à l'âge maternel de même que la fréquence (\%) des cas de syndrome de Down par rapport à d'autres anomalies chromosomiques peuvent être consultés dans l'annexe 3 .

Après étude cytogénétique des ovocytes humains, il s'avère que le pourcentage global d'anomalies chromosomiques, chromosomes surnuméraires ou sous-numéraires s'élève à $24 \%$, soit beaucoup plus qu'au niveau des spermatozoïdes. Un rôle majeur a été attribué au sexe féminin dans la genèse des anomalies chromosomiques de l'oeuf fécondé. Les données les plus significatives concernant la trisomie 21 dans la littérature sont liées à l'effet maternel : "Chez $80 \%$ des sujets atteints, le chromosome surnuméraire provient de la mère "(Pellestor F. 1990).

La spermatogenèse differe de l'ovogenèse par le renouvellement constant des spermatozoïdes, contrairement aux ovocytes stagnant dans l'ovaire dès le stade de 
l'enfance, ce qui peut provoquer un ovocyte vieillissant vers la fin de l'âge fertile. L'hypothèse formulée est basée sur le fait que l'ovogenèse débute par une très longue prophase méiotique et que les ovocytes produits à la fin de la vie génitale comportent une proportion plus élevée d'anomalies chromosomiques.

Une deuxième hypothèse suggère que l'âge maternel n'influe pas tant sur le processus de ségrégation chromosomique que sur la nidation et la survie « in utero » des foetus aneuploïdes. Par conséquent, la corrélation observée entre le vieillissement maternel et l'aneuploïdie résulterait d'une diminution de l'efficacité du mécanisme de sélection intra-utérin à l'égard des conceptus anormaux (Serre J.-L. et coll. 1983).

La signification de cette hypothèse est que dans le phénomène de la sélection naturelle, la plupart des malformations congénitales sont automatiquement éliminées dans un produit d'avortat. Cependant, les mères plus âgées rendent à terme les enfants trisomiques 21 car elles posséderaient un processus diminué d'élimination naturelle d'enfants anormaux.

Un troisième facteur étiologique possible mérite qu'on s'y attarde. Madame Andrée Cholette (médecin pathologiste au Centre hospitalier de Chicoutimi) propose que la gravité des séquelles physiques étant amoindrie dans la trisomie 21 comparativement avec les autres trisomies, la sélection naturelle qui rejette un embryon anormal dans un produit d'avortat n'intervient pas; ce qui a pour effet de rendre un foetus anormal à terme. 


\subsection{Gènes localisés sur le chromosome 21 associés au phénotype}

Cinq gènes, sans doute associés au phénotype de la trisomie 21, ont été localisés dans une petite région du bras long (q) du chromosome 21 (Patterson D. 1994). Ces gènes sont présentés dans la figure 8 .

Les gènes qui codent les protéines superoxyde dismutase (sod-1) et alpha-Acristalline sont situés sur les bandes 21 q22.1 et 21q22.3; les gènes Gart et ets-2 sont situés dans les bandes $21 \mathrm{q} 22.1$ et $21 \mathrm{q} 22.2$. Un gène qui code la phospho-fructokinase (pfkl) est également présent sur la bande 21q22.3.

A) Le gène identifié sur le chromosome 21 code la forme soluble de l'enzyme superoxydase dismutase (sod-1); ce sont des molécules très réactives libérées lors de l'oxydation et qui jouent sans doute un rôle dans le vieillissement. Les modifications de la concentration en sod-1 peuvent influencer le retard mental des personnes atteintes de trisomie 21 ainsi que dans leur vieillissement accéléré. Une équipe de chercheurs décrivent leur démarche scientifique qui a permis de localiser le gène (sod-1) sur le chromosome 21 (Sinet P.M. et coll. 1976).

B) Les cataractes et les défauts du cristallin que présentent les trisomiques 21 seraient dus à l'expression anormale de la protéine alpha-A-cristalline, un composant structurel du cristallin de l'oeil. 


\section{Figure 8}

Gènes associés au phénotype de la trisomie 21

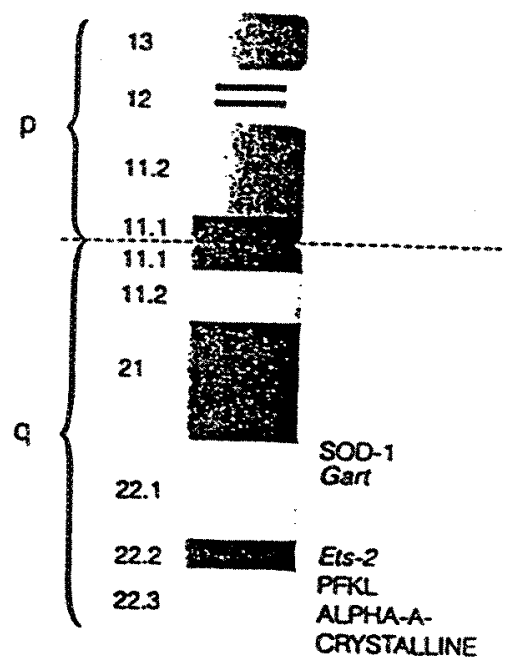

Source : Patterson David (1994). La trisomie 21. Dossier pour la science, avril, p. 5460. 
C) Le gène Gart qui code trois enzymes intervenant dans la synthèse des purines, explique l'aspect biochimique de la trisomie 21 . En effet; les enfants trisomiques 21 présentent une augmentation de la concentration en purines (deux des bases azotées qui entrent dans la composition de l'ADN et de l'ARN), ce qui cause des troubles neurologiques, un retard mental et des déficiences du système immunitaire. À cela s'ajoute un risque plus élevé de complications et un pronostic vital plus sévère. La trisomie de ce seul gène suffit à expliquer de nombreuses anomalies associées au syndrome de Down.

D) Le gène ets-2 est l'oncogène qui par son activité modifiée provoque la leucémie. Les médecins observaient depuis plusieurs années que les individus atteints de trisomie 21 devenaient souvent leucémiques. Les cancérologues ont cherché le mécanisme sousjacent à cette corrélation; ils ont montré qu'il existe un oncogène sur le chromosome 21 l'oncogène ets-2, et ils l'ont localisé sur la région chromosomique participant à la translocation entre les chromosomes 8 et 21 .

En 1985, Janet Rowley et ses collègues de l'Université de Chicago ont émis deux hypothèses : la leucémie serait provoquée par une modification de l'activité d'un ou de plusieurs oncogènes portés par le chromosome 21 ; la trisomie du chromosome 21 ou une translocation du matériel génétique du chromosome 21 sur le chromosome 8 serait responsable de cette modification (Patterson D. 1994). 
E) Je ne possède pas de données sur les impacts cliniques d'un enfant atteint de trisomie 21 concernant le gène qui code la phospho-fructokinase (pfkl).

Des chercheurs ont suggéré qu'il peut exister une relation génétique entre la maladie d'Alzheimer et la trisomie 21 (Farrar G. et coll. 1990). Ils ont démontré que la protéine amyloïde bêta, un des principaux constituants des plaques neurofibrillaires qui s'accumulent dans le cerveau des personnes atteintes de la maladie d'Alzheimer, est identique à la protéine qui s'accumule dans les lésions cérébrales apparemment semblables dans le cerveau de tous les trisomiques âgés de plus de 35 ans. Différents chercheurs ont localisé le gène codant la protéine amyloïde bêta sur le chromosome 21 . De plus, ils ont trouvé que le gène apparemment responsable d'une forme familiale de maladie d'Alzheimer se trouve également sur le chromosome 21 (Patterson D. 1994).

\subsection{Diagnostic prénatal}

Le diagnostic prénatal fait référence à l'ensemble des méthodes qui permettent l'approfondissement des connaissances sur l'état génétique et métabolique du foetus, sa constitution chromosomique (y compris le sexe) et ce, à un stade de plus en plus précoce de la grossesse (Durand S., Goulet C. 1992). Il permet des interventions précoces dans le respect des choix individuels. 
Les méthodes utilisées pour détecter la trisomie 21 sont l'amniocentèse, la biopsie choriale et la foetoscopie. L'amniocentèse est pratiquée non pas de façon systématique mais sous ordonnance médicale, en présence immédiate du médecin pathologiste.

Au Québec, la biopsie choriale ainsi que la foetoscopie sont actuellement utilisées dans certains centres hospitaliers des villes de Québec et de Montréal avec une certaine dose de prudence car les médecins pathologistes, non expérimentés, affinent leur technique de prélèvement dans le but de minimiser les risques inhérents à ces nouvelles pratiques.

Description des différentes méthodes utilisées pour établir un diagnostic prénatal de trisomie 21.

L'amniocentèse est la ponction du sac amniotique qui prélève de 10 à $20 \mathrm{ml}$ de liquide amniotique en vue d'une analyse pour la détection d'anomalies chromosomiques des cellules foetales et d'un examen biochimique. Elle est pratiquée habituellement entre la $15^{e}$ et la $16^{\mathrm{e}}$ semaine de grossesse.

La biopsie choriale consiste à prélever un fragment de tissu (villosités chorioniques) provenant de l'oeuf fertilisé, en insérant un instrument de biopsie à l'intérieur du col jusqu'à l'utérus. Elle s'effectue dès la $10^{\circ}$ semaine de grossesse. 
La foetoscopie est l'introduction d'un endoscope très fin par l'intermédiaire d'un trocart qui permet la visualisation directe du foetus, du cordon ombilical et de la face foetale des membranes du placenta $\left(15^{\circ}-16^{e}\right.$ semaine) et surtout le prélèvement de sang foetal $\left(18^{\mathrm{e}}-20^{\circ}\right.$ semaine) pour le diagnostic de certaines hémoglobinopathies. Le risque d'accident comme l'avortement et l'hémorragie foetoplacentaire est estimé à $5-10 \%$ (Fattorusso V., Ritter O. 1986).

Puisque l'amniocentèse est la méthode la plus couramment employée, d'autres données complètent sa description. L'amniocentèse comporte certains risques : elle entraîne dans là $2 \%$ des cas des hémorragies foetales ou maternelles et dans $0,3 \%$ des cas la perte du foetus.

Les principales indications de l'amniocentèse sont :

A) Grossesse chez une femme de plus de 38 ans.

B) Couple ayant eu un enfant atteint de trisomie.

C) Grossesse chez une femme conductrice saine d'une affection dont l'hérédité est liée au sexe, par exemple hémophilie A ou myopathie de Duchenne. La probabilité de la naissance d'un garçon atteint de la maladie étant de $50 \%$. La détection prénatale du sexe permet l'interruption de la grossesse si les parents le désirent.

D) Diagnostic d'un trouble métabolique du foetus. Une centaine d'anomalies héréditaires du métabolisme peuvent être détectées par l'amniocentèse. Celle- 
ci est indiquée lorsque les deux parents sont hétérozygotes (porteurs d'un gène autosomal récessif d'une anomalie du métabolisme).

E) Détermination du sexe du foetus. Étant donné les risques qu'elle comporte, l'amniocentèse n'est indiquée que si la mère est conductrice d'une anomalie génétique liée au sexe.

F) Détermination de l'alpha-foetoprotéine. L'indication principale de cette détermination est la naissance antérieure d'un enfant atteint d'un défaut de fermeture du tube neural (anencéphalie, spina bifida, etc.). En effet, l'augmentation de l'alpha-foetoprotéine est très spécifique de ces malformations, bien qu'on observe parfois aussi dans le syndrome de Turner, l'atrésie duodénale, la néphrose congénitale et les malformations multiples. Le sérum de la mère porteuse d'un enfant trisomique 21 est riche en foetoprotéine et en oestrogène (Wald $N$. J. et coll. 1988; Donnai D. et coll. 1988). La présence de sang dans le liquide amniotique peut fausser les résultats (Fattorusso V., Ritter O. 1986). Les indications de même que les facteurs de risque entre l'amniocentèse, la foetoscopie et la biopsie choriale sont présentées à l'annexe 6 .

Des statistiques au Québec démontrent l'ampleur croissante de la demande relative à ces méthodes. Même si les accouchements depuis 1979 ont diminué de $15 \%$, le nombre d'échographies obstétricales est passé de 67846 à 158222 entre 1979 et 1985 . Le nombre d'amniocentèses a doublé entre 1980 et 1986, passant de 1480 à 3141 . En 1985, 61 biopsies choriales ont été effectuées et on en dénombrait 139 au Québec en 1986. Entre 1979 et 1984, seulement 5 foetoscopies ont été exécutées au Québec (Durand S., Goulet C. 1992). 


\section{Dépistage systématique}

En France et aux États-Unis, le diagnostic prénatal pour la trisomie 21 s'effectue actuellement de façon systématique chez la femme enceinte de 35 ans et plus. La recherche des marqueurs de l'anomalie s'effectue par dosage des bêta HCG dans le sérum des femmes enceintes entre 15 et 18 semaines de grossesse (Petitnicolas C. 1994). Des auteurs s'interrogent sur l'âge maternel requis pour ce dépistage (Donnai D. et coll. 1988; McFadyen Iain R. 1988).

L'implication psychologique engendrée par l'annonce d'une telle anomalie suscite des controverses quant à l'utilisation des tests de dépistage systématiques chez les femmes enceintes de 35 ans et plus. "Outre les risques de multiplication abusive de tels examens, il existe aussi toute une série de problèmes liés à l'existence de faux positifs ou de faux négatifs » (Petitnicolas C. 1994).

Le docteur Ségolène Aymé, directrice du SC 11 de l'Inserm (consacré à la cartographie du génome humain à des fins de recherche clinique) commente le danger potentiel qu'un tel dépistage en masse soit fait en amateur. Elle suggère d'encadrer la disponibilité de ces tests par des recommandations voire même des réglementations (Petitnicolas C. 1994). 
CHAPITRE 3

MÉTHODOLOGIE 
Ce chapitre présente la collecte des données, les mesures démographiques, la construction des groupes témoins, l'étude généalogique des proposants et des groupes témoins, le calcul de la consanguinité (F) et le calcul d'apparentement (Phi) des proposants et des groupes témoins.

\subsection{Collecte des données}

Le diagnostic médical de la trisomie 21 se valide essentiellement par le résultat du caryotype qui prouve incontestablement la présence d'un chromosome surnuméraire sur la $21^{\mathrm{c}}$ paire. Le répertoire des caryotypes s'est effectué au département de pathologie du Centre hospitalier de Chicoutimi, celui-ci étant le seul département spécialisé dans toute la région du Saguenay Lac St-Jean à offrir l'instrumentation technologique nécessaire pour effectuer un caryotype ainsi que les cytogénéticiennes et les médecins pathologistes pour en interpréter fidèlement le résultat final.

Un instrument de mesure fut élaboré permettant la collecte de ces caryotypes, ainsi que d'autres données essentielles, dont les paramètres se décrivent comme suit :

- nom, prénom du proposant et no. dossier;

- date, mois et année de naissance du proposant;

- date à laquelle le caryotype a été effectué; 
- le sexe du proposant;

- le lieu de naissance du proposant;

- l'âge du père et de la mère du proposant;

- l'âge du proposant au moment du caryotype effectué;

- la configuration chromosomique du caryotype.

L'instrument de mesure pour la collecte des caryotypes est visualisé à l'annexe 4. Les informations décrites dans la section " données supplémentaires "permettaient de savoir le nombre de poussées cellulaires et de métaphases utilisées dans la technique, de même que l'interprétation finale du caryotype par le médecin pathologiste. Les recommandations du médecin pathologiste s'inscrivaient dans la section " commentaires ". L'instrument de mesure utilisé par le médecin demandant le caryotype est présenté en annexe 5.

\section{Collecte des caryotypes}

Dans le cadre de cette recherche, tous les caryotypes présents aux archives du Centre hospitalier de Chicoutimi ont été examinés un par un et chaque caryotype représentant une trisomie 21 a été sélectionné. De 1967 à 1990, les caryotypes définissant une trisomie 21 ont tous été répertoriés. Les 144 cas répertoriés représentent les enfants atteints du syndrome de Down dans la région du Saguenay Lac St-jean. 
Au cours de l'année 1967, le médecin pathologiste du Centre hospitalier de Chicoutimi de l'époque avait décidé d'examiner toutes les garderies existantes. II effectuait un caryotype à tous les enfants symptomatiques d'une trisomie 21 , quel que soit leur âge.

Il est certain que les caryotypes répertoriés de 1967 à 1974 ne représentent pas fidèlement la totalité des enfants atteints de trisomie 21 dans la région du Saquenay Lac St-Jean car une certaine proportion de médecins généralistes n'ont pas demandé le caryotype. Il ne faut pas oublier que durant cette période certains accouchements peuvent avoir eu lieu à domicile et que la symptomatologie d'un enfant atteint à sa naissance ait été trompeuse. Le reflet de cette réalité est qu'un enfant trisomique 21 ressemble étrangement à un phénotype d'enfant normal et que les symptômes de déficience intellectuelle ne se constatent que plus tard dans son évolution.

Cependant, les caryotypes d'enfants trisomiques 21 nés de 1975 à 1990 constituent quasiment l'ensemble de tous les enfants atteints de trisomie 21 nés durant cette période, d'une part grâce à l'implantation en 1974 d'une structure technologique extrêmement sophistiquée pour effectuer et interpréter fidèlement le résultat d'un caryotype, et du fait du suivi médical de tout nouveau-né par un pédiatre. Celui-ci, étant plus sensibilisé à cette pathologie particulière, s'assure d'un diagnostic médical valide avant d'annoncer aux parents l'état pathologique de leur enfant. 


\subsection{Données démographiques}

Après avoir répertorié les 144 caryotypes d'enfants atteints de trisomie 21 , différents instruments de mesure ont servi à acquérir les données essentielles à l'étude : l'identité du père et de la mère, leur lieu de résidence ainsi que la localité dans le Saquenay Lac St-Jean dans laquelle leur mariage a été célébré. Ces informations nous ont permis de jumeler ces données avec le fichier réseau BALSAC d'IREP (Bouchard G. et coll. 1991). Outre le lieu de résidence des proposants et des groupes témoins, ce fichier de population a permis de préciser ou de vérifier d'autres données démographiques : l'âge maternel et paternel, le mois, l'année de naissance ainsi que le sexe des proposants et des groupes témoins.

Quelques consultations auprès des presbytères dans les paroisses où certains couples s'étaient antérieurement mariés se sont avérées nécessaires pour compléter ces informations.

\section{Limites dans les données démographiques}

Pour un certain nombre de cas, les données démographiques n'ont pu être retracées :

- date de naissance imprécise ( mois ou année) du proposant; 
- erreur ou absence dans le prénom ou le nom d'un parent;

- père ou mère inconnu de l'enfant atteint;

- inexactitude du prénom de l'enfant qui ne s'appariait pas avec la configuration de ses gonosomes (chromosomes sexuels sur le caryotype).

Une première recherche des données nous a montré qu'au Lac St-Jean, il existait une différence parmi certaines de ces données entre le lieu de résidence des proposants et le centre hospitalier qui les recevait. Pour ces cas, nous avons effectué des recherches supplémentaires.

\section{Analyse des données démographiques}

L'impossibilité de préciser, ne serait-ce qu'une seule de ces données discordantes, a amené une première sélection parmi les 144 cas répertoriés.

Une deuxième sélection s'est effectuée, lorsque les parents ou les grands-parents des proposants ne s'étaient pas mariés au Saguenay Lac St-Jean. Nous ne pouvions conserver aucun de ces cas, vu l'impossibilité de poursuivre l'étude généalogique des proposants. 
Parmi les 144 cas répertoriés, 67 seulement ont participé à la reconstruction généalogique.

\subsection{Construction des groupes témoins}

Les trois groupes témoins reflètent le plus possible les paramètres de chaque proposant.

Définition du témoin de recherche

Cet enfant est supposé normal. L'année, le mois de sa naissance, le sexe et le lieu de la résidence de ses parents sont sensiblement similaires à l'enfant trisomique 21 .

Chaque proposant (67) a été apparié avec trois groupes témoins, ce qui fait 201 témoins de recherche.

Pour la période de 1971 à 1986, le fichier sectoriel du BSQ d'IREP (Guérin, M. 1990) a facilité la recherche parmi la population cible de ces témoins. 
Les noms et prénoms des enfants du même sexe que le proposant dont les parents résidaient dans la même localité au Saguenay Lac St-Jean et qui de plus naissaient la même année que le proposant, représentent les critères de sélection du groupe contrôle.

Une des difficultés était de répertorier le mois de naissance le plus représentatif du proposant. Pour certains cas, nous n'avons pas tenu compte du mois de naissance étant donné la faible taille des effectifs dans certaines municipalités. Nous avons donc réajusté avec l'équipe d'IREP le programme informatisé qui permettait d'élargir ce paramètre.

\section{4 Étude généalogique des proposants et des groupes témoins}

La reconstruction généalogique ascendante des 67 proposants ainsi que des 201 témoins de recherche du groupe contrôle s'est effectuée sur une profondeur de cinq générations.

L'IREP dispose d'un fichier de population informatisé (fichier réseau BALSAC) contenant 660000 actes de l'état civil du Saguenay (baptêmes, mariages et sépultures), couvrant la période 1842-1971. C'est à l'aide de ces données que sont produites par ordinateur les généalogies ascendantes (Bouchard G. et coll. 1986, doc. 111-C-49). 


\subsection{Calcul de la consanguinité (F) et de l'apparentement (Phi) des proposants et des groupes témoins}

Le coefficient de consanguinité (f) vise à identifier les ancêtres communs du père et de la mère et par la suite définir sous la forme d'une probabilité, la possibilité que cet ancêtre commun ait effectivement transmis les deux copies d'un même gène à un locus donné sur le chromosome (l'un venant de la mère, l'autre venant du père). Ce coefficient désigne la probabilité pour une personne (=EGO) de porter à un locus donné deux copies d'un même gène. Le cas échéant, il s'ensuit que les parents de EGO ont dû recevoir ces copies d'un ancêtre commun plus ou moins éloigné. La recherche généalogique ascendante vise à identifier cet ancêtre et à exprimer sous la forme d'une probabilité la possibilité qu'il ait effectivement transmis les deux copies du gène.

Le coefficient d'apparentement (Phi) désigne la probabilité que deux individus portent à un même locus, chacun une copie d'un même gène. La recherche généalogique ascendante parmi les individus (père-mère) permet le calcul de la probabilité que ces mêmes individus portent à un même locus, chacun une copie d'un même gène. Dans ce dernier cas, le coefficient de parenté du couple est égal au coefficient de consanguinité de leurs enfants puisque la probabilité que deux individus portent chacun une copie d'un même gène à un locus et la probabilité qu'ils la transmettent tous deux à leurs descendants sont identiques (Bouchard G. et coll. 1986, doc. 111-C-46). 
Les recherches généalogiques ascendantes ont permis le calcul du coefficient de consanguinité (f) et du coefficient de parenté (phi) des 67 proposants et des 201 témoins de recherche, de même que la recherche de fondateurs communs.

\subsection{Considérations éthiques}

Nous avons respecté fidèlement les procédures concernant la confidentialité des dossiers répertoriés aux archives du Centre hospitalier de Chicoutimi. Chaque dossier répertorié a été assujetti à une identification numérique dans le but de conserver cette confidentialité. 
CHAPITRE 4

RÉSULTATS 
Ce chapitre présente les résultats obtenus après avoir effectué une recherche épidémiologique et génétique sur la trisomie 21.

\subsection{Configurations chromosomiques}

Parmi les 144 cas répertoriés, l'on retrouve plusieurs configurations chromosomiques différentes représentant le phénotype du syndrome de Down. Le tableau 1 indique cette fréquence.

Tableau 1

Fréquence des configurations chromosomiques

\begin{tabular}{||l|c|}
\hline \multicolumn{1}{|c|}{ CARYOTYPE } & FREQUENCE \\
\hline $47, \mathrm{XX}$ ou $\mathrm{XY},+21$ & 135 \\
\hline $46, \mathrm{XX}$ ou $\mathrm{XY} / 47, \mathrm{XX}$ ou $\mathrm{Xy},+21$ & 3 \\
\hline $46, \mathrm{XY} / 46, \mathrm{XY},+21,-\mathrm{C} / 47, \mathrm{XY},+21$ & 1 \\
\hline $47, \mathrm{XY},+21 / 48, \mathrm{XY},+21,+21$ & 1 \\
\hline $47, \mathrm{XX},+21 / 47, \mathrm{XY},+21$ & 1 \\
\hline $46, \mathrm{XY},+\mathrm{t}(14 ; 21)(\mathrm{p} 11 ; \mathrm{q} 11)$ & 1 \\
\hline $46, \mathrm{XX},-21,+\mathrm{t}(21 \mathrm{q} 21 \mathrm{q})$ & 2 \\
\hline TOTAL & 144 \\
\hline
\end{tabular}

Voir la définition de la nomenclature aux pages 20 et 32 . 
4.2 Calcul de la consanguinité et de l'apparentement des proposants et des groupes témoins

Le coefficient moyen de consanguinité est plus élevé dans le groupe trisomique 21 que dans le groupe contrôle $(13,4824$ versus 8,4781$)$. Un test de distribution at de Student a été effectué et démontre que cette différence n'est pas statistiquement significative $(\mathrm{P}>0,10)$.

Ces résultats sont présentés dans le tableau 2. L'entropie est une mesure de la profondeur des généalogies (quantité d'information disponible dans un système) (Kouladjian K. J. 1986).

Tableau 2

Coefficient moyen de consanguinité (F), coefficient de parenté (Phi) et entropie des échantillons

\begin{tabular}{|l|c|c|c|c||}
\hline & Nombre de cas & $\begin{array}{c}\text { Consanguinité } \\
\mathbf{x 1 0 - 4}\end{array}$ & $\begin{array}{c}\text { Parenté } \\
\mathbf{1 0 ~ 4}\end{array}$ & $\begin{array}{c}\text { Entropie } \\
\text { (var) }\end{array}$ \\
\hline Down & 67 & 13,4824 & 2,2175 & $4,17(0,82)$ \\
\hline Témoins 1 & 67 & 4,9557 & 1,6676 & $4,26(0,97)$ \\
\hline Témoins 2 & 67 & 6,4132 & 1,6977 & $4,27(1,07)$ \\
\hline Témoins 3 & 67 & 14,0654 & 1,5834 & $4,24(0,95)$ \\
\hline $\begin{array}{l}\text { Moyenne } \\
\text { Témoins }\end{array}$ & & 8,4781 & 1,6496 & \\
\hline
\end{tabular}

(F) Source : Programme MEDIC 4, IREP (Bouchard G., De Braekeleer M. et coll. 1991). (PHI) Source : Programme APPB, IREP (Bouchard G., De Braekeleer M. et coll. 1991). 
Le coefficient d'apparentement du groupe trisomique 21 est légèrement augmenté par rapport à celui du groupe témoin $(2,2175$ versus 1,6496$)$, mais le résultat n'est pas statistiquement significatif $(\mathrm{P}>0,10)$. Cette augmentation est due à la relation 1:2 qui existe entre deux proposants.

\subsection{Effet fondateur}

La reconstruction généalogique sur une profondeur de cinq générations a permis de constater qu'il n'y a pas plus d'ancêtres communs chez les proposants que chez les groupes témoins. II n'y a donc pas d'effet fondateur chez la trisomie 21. Le nombre de couples qui apparaissent plus qu'une fois (occurrence) est présenté dans le tableau 3.

Tableau 3

Occurrence des fondateurs

\begin{tabular}{|c|r|r|r|r|}
\hline $\begin{array}{c}\text { Couples } \\
\text { Occurrence }\end{array}$ & Down & Témoins 1 & Témoins 2 & Témoins 3 \\
\hline 2 & 148 & 132 & 141 & 161 \\
\hline 3 & 46 & 39 & 38 & 22 \\
\hline 4 & 9 & 16 & 16 & 15 \\
\hline 5 & 3 & 4 & 4 & 6 \\
\hline 6 & 0 & 5 & 5 & 2 \\
\hline 7 & 0 & 2 & 2 & 0 \\
\hline
\end{tabular}

Source : IREP 


\subsection{Calcul de la prévalence}

De 1975 à 1990 , la prévalence d'enfants trisomiques 21 est de $1 / 1219,5$. Le tableau 4 présente ces résultats.

Tableau 4

Calcul de la prévalence des trisomiques 21 de 1975 à 1990

\begin{tabular}{|c|c|c|}
\hline Année de naissance & Fréquence & Nombre de naissances \\
\hline 1975 & 3 & 4924 \\
\hline 1976 & 5 & 5309 \\
\hline 1977 & 3 & 5209 \\
\hline 1978 & 3 & 5439 \\
\hline 1979 & 6 & 5808 \\
\hline 1980 & 5 & 5610 \\
\hline 1981 & 8 & 5386 \\
\hline 1982 & 3 & 4957 \\
\hline 1983 & 2 & 4707 \\
\hline 1984 & 3 & 4592 \\
\hline 1985 & 3 & 4324 \\
\hline 1986 & 4 & 3981 \\
\hline 1987 & 3 & 3769 \\
\hline 1988 & 7 & 3737 \\
\hline 1989 & 2 & 3842 \\
\hline 1990 & 2 & 4027 \\
\hline TOTAL & 62 & 75621 \\
\hline PRÉVALENCE & & $1 / 1219,5$ \\
\hline
\end{tabular}

Sources : CLSC du Saguenay Lac St-Jean (1975-1979); BSQ/Région 02 (1980-1990) 
Parmi les 144 cas de trisomie 21 répertoriés dont les années de naissance se situent de 1940 à 1991, seules les naissances de 1975 à 1990 (62) représentent cet échantillon.

\subsection{Fréquence de la trisomie 21 dans les localités du Saguenay Lac St-Jean}

Le tableau 5 présente la fréquence d'enfants atteints de la trisomie 21 dans la région du Saguenay ainsi que les sous-régions du Lac St-Jean.

Tableau 5

Fréquence de la trisomie 21 par localités au Saguenay Lac St-Jean (1975 à 1990)

\begin{tabular}{|l|c|l|c|}
\hline \multicolumn{1}{|c|}{ Lieu de résidence } & Fréquence & Lieu de residence & Fréquence \\
\hline Albanel & 3 & Métabetchouan & 1 \\
\hline Alma & 8 & Mistassini & 2 \\
\hline Anse St-Jean & 1 & Normandin & 2 \\
\hline Chambord & 1 & Petit Saguenay & 1 \\
\hline Chicoutimi & 8 & St-Ambroise & 2 \\
\hline Dolbeau & 1 & St-Félicien & 1 \\
\hline Ferland-Boileau & 1 & St-Fulgence & 1 \\
\hline Jonquière & 11 & St-Gédéon & 1 \\
\hline La Baie & 5 & St-Thomas Didyme & 3 \\
\hline La Doré & 1 & Inconnu & Total \\
\hline Larouche & 1 & & 56 \\
\hline
\end{tabular}

Source : IREP 
Parmi les 62 proposants qui sont nés de 1975 à 1990, six proposants sont absents de cet échantillon car ils résidaient dans les municipalités avoisinantes du Saguenay Lac St-Jean (Sacré-Coeur, Les Escoumins et Chibougamau).

\subsection{Moyenne d'âge maternel et paternel des proposants et des groupes témoins}

Le tableau 6 indique la moyenne et la variance de l'âge maternel et paternel des proposants et des groupes témoins.

Tableau 6

Moyenne et variance de l'âge maternel et paternel des proposants et des trois groupes témoins

\begin{tabular}{|l|l|c|c|c|}
\hline \multicolumn{1}{c|}{} & $\begin{array}{c}\text { Syndrome de } \\
\text { Down }\end{array}$ & Témoins 1 & Témoins 2 & Témoins 3 \\
\hline Âge mère & 35,4 (40 cas) & 27,6 & 28,8 & 27,4 \\
\hline Variance & 10,68 & 6,48 & 5,52 & 15,7 \\
\hline \hline Âge père & 37,7 (38 cas) & 32,0 & 31,1 & 30,1 \\
\hline Variance & 10,95 & 25,7 & 2,81 & 5,11 \\
\hline
\end{tabular}

Source : IREP

La moyenne de l'âge maternel des proposants $(35,4)$ est supérieure à la moyenne de l'âge maternel des trois groupes témoins $(27,9)$. 
La moyenne de l'âge paternel des proposants $(37,7)$ est également supérieure à la moyenne de l'âge paternel des trois groupes témoins $(31,07)$.

4.7 Fréquence des enfants atteints de trisomie 21 selon le mois de leur naissance Le tableau 7 présente le mois de naissance de tous les enfants trisomiques 21 répertoriés (144 cas).

Tableau 7

Fréquence des enfants trisomiques selon le mois de naissance

\begin{tabular}{||c|c|}
\hline Mois de naissance & Nombre d'enfants trisomiques \\
\hline Janvier & 11 \\
\hline Février & 9 \\
\hline Mars & 10 \\
\hline Avril & 15 \\
\hline Mai & 17 \\
\hline Juin & 17 \\
\hline Juillet & 13 \\
\hline Août & 10 \\
\hline Septembre & 10 \\
\hline Octobre & 9 \\
\hline Novembre & 10 \\
\hline Décembre & 11 \\
\hline Inconnus & 2 \\
\hline
\end{tabular}


Ces résultats indiquent un taux un peu plus élevé d'enfants atteints en avril, mai et juin.

\subsection{Rapport de masculinité des proposants}

Le calcul du Khi-deux a démontré que le sexe masculin domine par rapport au sexe féminin $X^{2}=5,17(P<0,05)$. Ce résultat est statistiquement significatif. Le tableau 8 nous montre cette prédominance masculine.

\section{Tableau 8}

Fréquence des proposants selon le sexe

\begin{tabular}{|c|c|c|}
\hline FEMME & HOMME & TOTAL \\
\hline 57 & 84 & 141 \\
\hline
\end{tabular}

Ce calcul représente tous les cas de trisomie 21 répertoriés (144 cas). Trois proposants dont les configurations chromosomiques étaient douteuses pour les gonosomes sont absents de cet échantillon. 
CHAPITRE 5

DISCUSSION ET RECOMMANDATIONS 
Ce chapitre présente les interprétations de chaque résultat, suscitant une panoplie de questionnements et de réflexions.

La fréquence des configurations chromosomiques différentes dans les caryotypes répertoriés corroborent le pourcentage décrit dans la littérature. En effet, nous retrouvons un nombre élevé de trisomies 21 franches et un pourcentage moindre de trisomies 21 acquises par translocation et par mosaïcisme.

Le coefficient moyen de parenté du groupe trisomique 21 est légèrement augmenté par rapport à celui du groupe témoin. Ce résultat s'explique par la relation 1:2 qui a existé entre deux proposants.

Le coefficient de consanguinité est plus élevé dans le groupe trisomique 21 que le groupe contrôle. Ce résultat, n'étant pas statistiquement significatif, ne corrobore pas tout à fait l'étude réalisée au Koweit où le taux de consanguinité des enfants trisomiques 21 est significativement plus élevé que celui du groupe contrôle (Alfi O.S. et coll. 1980). Ce résultat ne corrobore pas non plus la recherche effectuée en Turquie (Basaran N. et coll. 1992), de même que celle effectuée en Espagne (Martinez-Frias M.L. 1993), où l'on retrouve un même taux de consanguinité chez les trisomiques 21 que chez le groupe contrôle. 
Cependant les résultats obtenus sont similaires à la recherche effectuée dans les Iles Shetland où le coefficient de consanguinité des trisomiques 21 est plus élevé que celui du groupe contrôle, mais de façon non significative et où le coefficient d'apparentement des trisomiques 21 est le même que celui du groupe témoin (Roberts D.F. et coll. 1991). Cet auteur postule qu'un gène autosomal récessif peut être impliqué chez les homozygotes, possiblement en prévenant la perte des foetus trisomiques 21 . Cette hypothèse formulée peut nous sensibiliser sur le rôle de la génétique influençant la trisomie 21 chez les mères plus jeunes en comparaison avec les mères plus âgées où d'autres facteurs jouent un rôle.

Plusieurs recherches ont été effectuées sur la consanguinité des enfants atteints de trisomie 21 et les postulats sur l'hypothèse de gène autosomal récessif differrent; les uns acceptent l'hypothèse, les autres la rejettent. Les résultats de cette recherche tendent à favoriser cette hypothèse du fait de la sélection des groupes témoins dans une région qui par sa situation géographique favorise un certain degré d'apparentement et d'unions préférentielles.

Le résultat de la prévalence obtenue $1 / 1219,5$ ne correspond pas au taux d'incidence observé dans la littérature (1/700 à 1000 naissances). Ce résultat n'est qu'une sous-estimation de la prévalence. Même si les pédiatres depuis 1974 demandent systématiquement un caryotype à chaque naissance d'un enfant symptomatique de trisomie 21, il y a certainement des cas qui n'ont pas été recensés pour diverses raisons : décès en bas âge, accouchement à domicile et enfant caché par la honte de ses parents. 
Le résultat de la fréquence de la trisomie 21 par localités au Saguenay Lac St-Jean ne peut être interprété compte tenu du découpage géographique très fin (petit nombre d'effectifs dans les localités). Les localités où la fréquence est plus élevée sont celles où la population est également plus nombreuse.

La moyenne de l'âge maternel du groupe trisomique 21 est supérieure à la moyenne de l'âge maternel du groupe contrôle. Ce résultat corrobore les hypothèses formulées dans la littérature concernant les facteurs de risques associés à l'âge maternel avancé.

La fréquence de la trisomie 21 selon le mois de naissance indique un taux légèrement plus élevé pour les mois d'avril, mai et juin. Ce résultat est comparable à l'ensemble des naissances. En effet, dans les données du B.S.Q., le nombre des naissances est plus élevé au printemps par rapport à d'autres saisons durant l'année.

Dans cette étude, le sexe masculin prédomine par rapport au sexe féminin. Ce résultat est similaire à ceux des différentes recherches effectuées sur la trisomie 21 décrites dans la littérature. En effet, nous retrouvons constamment dans l'échantillonnage de ces recherches un nombre plus élevé d'hommes que de femmes trisomiques 21. 


\section{RECOMMANDATIONS}

L'étude de la pathologie globale du syndrome de Down et de ses conséquences a permis d'observer certains aspects humains, d'où l'intérêt de proposer certaines suggestions pratiques.

Une entrevue réalisée avec la responsable du service de maternité du Centre hospitalier de Chicoutimi m'a permis de connaitre la structure et la démarche de relation d'aide et de soutien aux parents qui ont à prendre une décision fort émotive; celle de mettre à terme ou non leur enfant diagnostiqué trisomique 21. Pour les intervenants en santé auprès de ce couple en difficulté, il m'apparaît essentiel qu'ils se familiarisent avec les interventions favorisant la prise de décision et le processus de résolution de la perte d'un enfant.

Cette équipe multidisciplinaire de soutien aux parents s'avère indispensable à mon avis compte tenu que l'âge de grossesse requis pour l'amniocentèse se situe entre 14 et 18 semaines et que le résultat de cet examen n'est remis qu'un mois plus tard aux parents. Ajoutons à cela le délai de la réaction émotive des parents à l'annonce d'un résultat positif de trisomie 21 de leur enfant et nous obtenons un avortement thérapeutique de 26 semaines. 
Cependant, à ce stade de la grossesse nous ne parlons plus d'avortement mais d'un accouchement provoqué; ce qui accroît à mon avis les conséquences psychiques d'un couple face à la prise de décision et au deuil de leur enfant. Pour toutes ces considérations, je propose une réflexion médicale concernant l'amniocentèse. Cet examen pourrait peut-être s'effectuer plus tôt et le résultat remis aux parents serait anticipé par rapport au délai actuel.

L'étude de la méthode californienne de stimulation cérébrale permettant à un enfant atteint de trisomie 21 d'acquérir une plus grande autonomie, représente pour des parents une mesure rigoureuse et difficilement applicable dans la réalité d'un couple. Cependant, elle redonne une certaine dose d'espoir à un enfant atteint : effectuer quelques activités quotidiennes comme sa soeur ou son frère. 
CONCLUSION 
La méthodologie utilisée pour cette recherche s'est avérée efficace pour établir un diagnostic fidèle de trisomie 21. Les caryotypes se sont révélés une source d'information précise pour atteindre l'objectif visé. De plus, le fichier de population informatisé d'IREP a permis l'identification d'une multitude d'éléments touchant la spécificité de cette pathologie.

Cette recherche a démontré que les facteurs de consanguinité et d'apparentement du groupe trisomique 21 ne présentent pas de différence statistiquement significative par rapport au groupe contrôle.

Les entrevues avec les intervenants en santé ont démontré l'importance d'être sensibilisé à l'aspect psychosocial de cette pathologie; d'une part pour l'enfant atteint qui subit la lourdeur des perceptions sociales et d'autre part, pour le couple qui est confronté à un diagnostic prénatal de trisomie 21 . 


\section{BIBLIOGRAPHIE}


Alfi O.S., Chang Rohnu, Azen Stanley P. (1980). Evidence for Genetic Control of Nondisjunction in Man. American Society of Human Genetics, vol. 32, p. 477-483.

Antonarakis S. E., Hassold T.J., Jacobs P.A., Petersen M.B. (1991). Origine parentale du chromosome surnuméraire dans la trisomie 21. Médecine/Sciences, France: John Libbey Eurotext, vol. 7, p. 520-521.

Arenson Edward B., Forde Diane (1989). Bone marrow transplantation for acute leukemia and Down syndrome : Report of a successful case and results of a national survey. The Journal of Pediatrics, vol. 114, p. 69-72.

Baird Patricia A., Sadovnick Adele D. (1988). Life expectancy in syndrome adults. Epidemiology. The Lancet, december 10, p. 1354.

Basaran N., Cenania A., Sayli B.S., Ozkinay C., Artan S., Seven H., Basaran A., Dinçer S. (1992). Consanguineous marriages among parents of Down patients. Clin. Genet., vol. 42, p. 13-15.

Bott C.E., Sekhon G.S., Lubs H.A. (1975). Unexpected high frequency of paternal origin of trisomy 21. Hum. Genet., october, Atlas, p. 236.

Bouchard G., Gradie M.I., Kouladjian K. (1986). Note sur les concepts relatifs à la consanguinité et à la parenté en langue française et en langue anglaise. IREP (Programme de recherches en génétique humaine), document no.: III-C-46, 3 pages.

Bouchard G., De Braekeleer M. (1991). Histoire d'un génome. Presses de l'Université du Québec, 607 pages. 
Bouchard G., Kouladjian k. (1986). Epidémiologie génétique à l'aide d'un fichier de population : développement d'un système d'inférence généalogique. IREP (Programme de recherches en génétique humaine), document no.: 111-C-49, 17 pages.

Brown Frank R, Greer Margaret K., Aylward Elizabeth H., Hunt Hurshell H. (1990). Intellectual and Adaptive Functioning in Individuals With Down Syndrome in Relation to Age and Environmental Placement. Pediatrics, vol. 85, p. 450-452.

Churchill Larry R. (1989). Bone marrow transplantation, physician bias, and Down syndrome : Ethical reflections. The Journal of Pediatrics, January, p. 87-88.

De Braekeleer M. (1989). Épidémiologie génétique II. Cours académique.

De Grouchy J., Turleau C. (1977). Atlas des maladies chromosomiques. Paris : Expansion scientifique francaise, 356 pages.

Donnai Dian, Andrews Tony (1988). Screening for Down's syndrome. BMJ, october, vol. 297, p. 876.

Dreyfus J-C. (1985). Chromosomes paternels et maternels. Médecine/Sciences, France; John libbey Eurotext, vol. 1, p. 336.

Durand Suzanne, Goulet Céline (1992). Le diagnostic prénatal. Mieux comprendre pour mieux agir. Nursing Québec, vol. 12, p. 15-24. 
Farrar Gillian, Altman Paul, Welch Simon, Wychrij Oryst, Ghose Bharati, Lejeune Jérome, Corbett John, Prasher Vee, Blair John A. (1990). Defective gallium-transferrin binding in Alzheimer disease and Down syndrome : possible mechanism for accumulation of aluminium in brain. The Lancet, vol. 335, p. 747-750.

Fattorusso V., Ritter O. (1986) . Vademecum clinique du symptôme à l'ordonnance. Italie, Masson, $11^{\mathrm{e}}$ édition, 1661 pages.

Giraud F., Mattei J.F. (1975). Aspects épidémiologiques de la trisomie 21. Hum. Genet., vol. 23, p. 1-30. Atlas, p. 242.

Gompertz Janet (1990). Improving communication in Down syndrome. The Lancet, may, p. 1278.

Green Jennifer (1988). Hepatitis B and Down's syndrome. BMJ, vol. 297, november, p. 1336.

Guérin Michel (1990). Étude descriptive du fichier des naissances, mariages et décès (1997-1986) du bureau de la Statistique du Québec. IREP, document no.: 1-C-98, 46 pages.

Hara Y., Sasaki M. (1975). A note on the origin of extra chromosomes in trisomies 13 and 21. Proc. Japan Acad. vol. 51, p. 295-299.

Hook B. (1981). Rates of Chromosome abnormalities at Different Maternal Age. Obstetrics \& Gynecology, vol. 58, p. 282-285. 
Huret J.L., Rahmani Z., McCormick M.K., Korenberg J. (1990). La plus petite région du chromosome 21 dont la triplication est nécessaire pour provoquer le phénotype du mongolisme. Médecine/Sciences, France: John Libbey Eurotext, décembre, vol. 6, p. 1025-1026.

Kjessler B., La Chapelle A. (1971). Meiosis and spermatogenesis in two post- pubertal males, with Down's syndrome: 47,XY,G+. Clin.Genet., vol. 2, p. 50-57. Atlas, p. 242.

Kouladjian Kevork (1986). Une mesure d'entropie généalogique. IREP (Programme de recherches en génétique humaine), document no.: 111-C-43, 4 pages.

Lilienfeld A.M., Benesch C.H. (1969). Epidemiology of mongolism. Baltimore, The Johns Hopkins Press, Atlas, p. 236.

Martinez-Frias M.L. (1993). Consanguineous marriages among parents of patients with Down syndrome. Clin. Genet. vol. 44, p. 221-222.

Mastroiacovo P., Bertollini R., Corchia C. (1990). Survival trends in Down syndrome. The Lancet, vol. 335, may, p. 1278-1279.

McFadyen Iain R. (1988). Screening for Down's syndrome. BMJ, vol. 297, november, p. 1335 .

Mikkelsen M. (1972). The effect of maternal age on the incidence of Down's syndrome. Hum. Genet., vol. 16, p. 141-146. Atias, p. 236.

Patterson David (1994). La trisomie 21. Dossier pour la Science, avril, p. 54-60. 
Pellestor Frank (1990). Étude cytogénétique de l'ovocyte humain. Médecine/Sciences, France: John Libbey Euroyext, décembre, vol. 6, p. 966-970.

Penrose L.S. (1933). The relative effects of paternal and maternal age in mongolism. Atlas, p. 236.

Petitnicolas Catherine (1994). Le mongolisme au carrefour du dépistage. La Presse, Montréal, octobre, p. 4.

Prieur M. (1968). Etude statistique du quotient intellectuel de 474 enfants atteints de trisomie 21. Thèse Doctorat Medecine, Paris, vol. 29, Atlas p.240.

Rethore M.O., Lafourcade J., Prieur M., Caille B., Cruveiller J. (1970). Mère et fille trisomiques 21 libres. Ann. Génét. vol. 13, p. 42-45. Atlas, p. 240.

Roberts D.F., Roberts M.J., Johnston A.W. (1991). Genetic epidemiology of Down's syndrome in Shetland. Hum. Genet., vol. 87, p. 57-60.

Scharrer S., Stengel-RutKowski S., Rodewald-Rudescu A., Erdlen E., Zang K.D. (1975). Reproduction in a female patient with Down's syndrome. Case report of a $46, \mathrm{XY}$ child showing slight pnenotypical anomalies, born to a $47, \mathrm{XX},+21$ mother. Hum. Genet., vol. 26, p. 207-214. Atlas, p.240.

Schneider Daniel, Zahka Kenneth G., Clark Edward B., Neill Catherine A. (1989). Patterns of Cardiac Care in Infants With Down Syndrome. AJDC, vol. 143, p. 363-365. 
Serre J.-L., Feingold J., Gallano P., Boué J., Boué A. (1983). Maintien et évolution des fréquences des anomalies de structures chromosomiques : application à quelques anomalies étudiées chez l'homme. Population 2, p. 283-306.

Sinet P.M., Couturier J., Dutrillaux B., Poissonnier M., Raoul O., Rethore M.O., Allard D., Lejeune J., Jerome H. (1976). Trisomie 21 et superoxyde dismutase. Tentative de localisation sur la sous-bande 21q22.1. Atlas, p. 234.

Solomon E. P., Davis P. W. (1981) . Anatomie et Physiologie humaine. Adaptation française Cholette C. Montréal, McGraw-Hill, 668 pages.

Thompson Ian M., Thompson Donna D. (1988). Male fertility and the undescended testis in Down syndrome. PGM, october, vol. 84, p. 299, 302-303.

Wald Nicholas J., Cuckle Howard S., Densem James W., Nanchahal Kiran, Royston Patrick, Chard Tim, Haddow James E., Knight George J., Palomaki Glenn E., Canick Jacob A. (1988). Maternal serum screening for Down's syndrome in early pregnancy. BMJ, october, vol. 297, p. 883-887. 
ANNEXES 


\section{ANNEXE 1}

Représentation des lieux géographiques de la population cible : la région du Saguenay et ses sous-régions

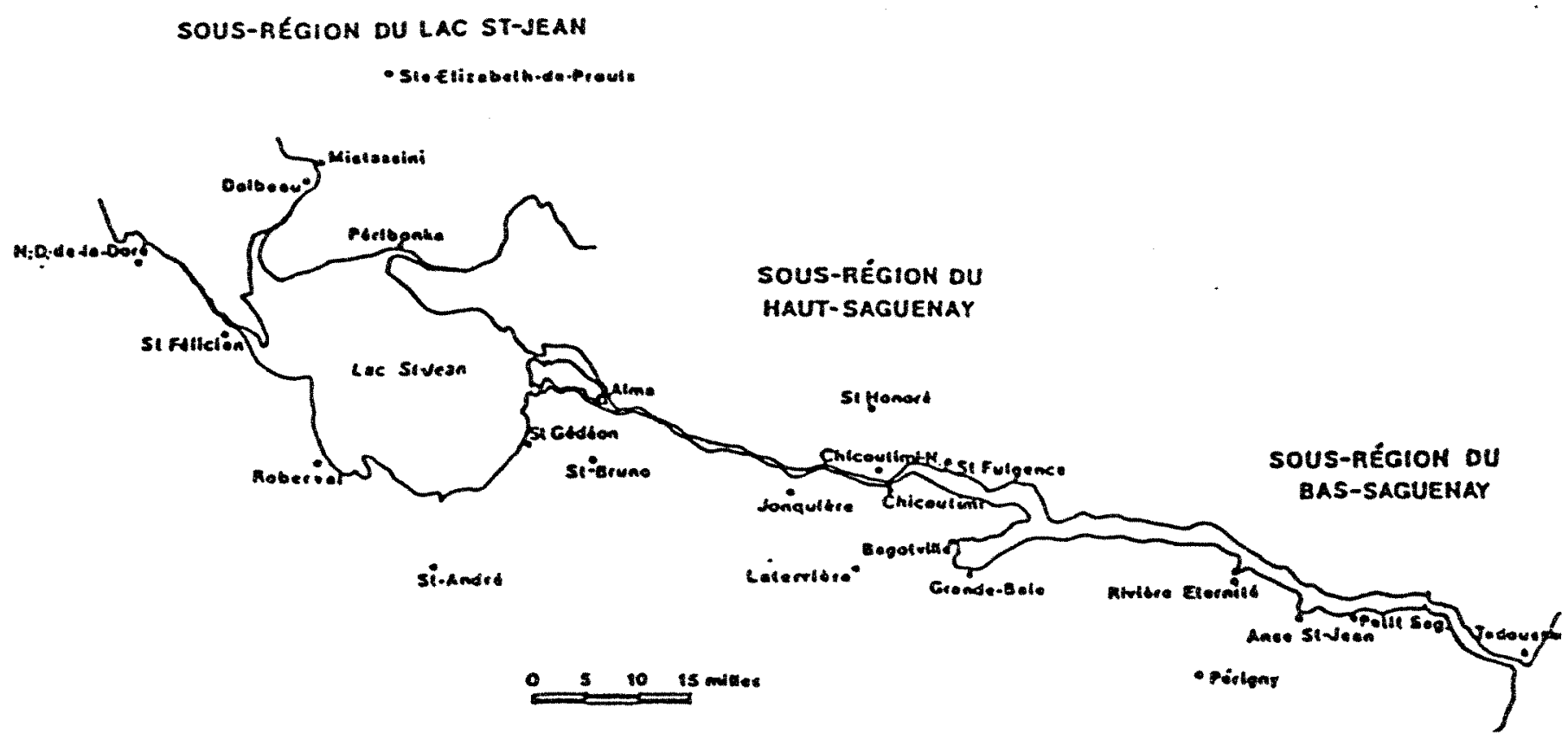

Source : IREP 


\section{ANNEXE 2

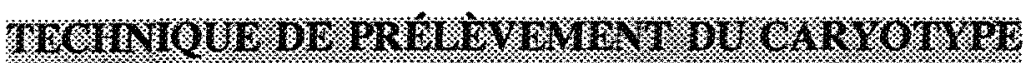 \\ Renseignements relatifs à une demande \\ d'examen cytogénétique au Centre hospitalier de Chicoutimi}

\section{Tenps de preirenenent}

- À jeun le matin ou le patient étant à jeun depuis au moins 2 heures.

- Le mercredi, jeudi ou vendredi; de préférence le mercredi pour les hôpitaux extérieures afin d'éviter que le prélèvement ne reste en entrepôt ou au bureau de poste.

\section{Techingue de prelevenent}

- Désinfecter la peau avec de l'éthanol à $70 \%$.

- Prélever par ponction veineuse de 2 à $5 \mathrm{ml}$ de sans dans une seringue contenant de l'héparine à raison de 50 unités par $\mathrm{ml}$ de sang (héparine à $1000 \mathrm{u} / \mathrm{ml}$ ).

$2 \mathrm{ml}$ de sang $\quad 0,1 \mathrm{ml} \mathrm{d}$ 'héparine

$3 \mathrm{ml}$ de sang $\quad 0,15 \mathrm{ml}$ d'héparine

$4 \mathrm{ml}$ de sang $\quad 0,2 \mathrm{ml}$ d'héparine

$5 \mathrm{ml}$ de sang $\quad 0,25 \mathrm{ml} \mathrm{d}$ 'héparine

- Enlever l'aiguille et mettre le sang dans un tube stérile tel que le tube Falcon no. 2003.

- Faire parvenir le prélèvement le même jour par courrier «livraison spéciale» ou par transport (note : avertir le laboratoire de tout envoi).

- Déposer le tube dans un thermos ou boîte «styrofoam» préalablement réfrigéré, ouvert, durant 24 heures.

\section{Remarques}

Il est préférable que le patient n'ait reçu aucune radiation ionisante ni médicament antimitotique au moins un mois avant son examen.

Source : Laboratoire de cytogénétique

Département de pathologie /Centre hospitalier de Chicoutimi 
ANNEXE 3

RISQUES ASSOCIÉS À L'ÂGE MATERNEL, DE MÊME QUE LA FRÉQUENCE (\%) DU SYNDROME DE DOWN PAR RAPPORT À D'AUTRES ANOMALIES

\begin{tabular}{|c|c|c|c|}
\hline MATERNEL & $\begin{array}{l}\text { SYNDROME } \\
\text { DE DOWN } \\
\text { Moyerne/1000) }\end{array}$ & DE RISQUE & $\begin{array}{l}\text { SVNDROME DE DOWN } \\
\text { \% PAR RAPPORT AUX AUTRES ANOMALIES }\end{array}$ \\
\hline 20 & 0,6 & 0,6 & 32 \\
\hline 21 & 0,6 & 0,6 & 32 \\
\hline 22 & 0,7 & 0,7 & 35 \\
\hline 23 & 0,7 & 0,7 & 35 \\
\hline 24 & 0,8 & 0,8 & 38 \\
\hline 25 & 0,8 & 0,8 & 38 \\
\hline 26 & 0,85 & 0,9 & 40 \\
\hline 27 & 0,9 & 0,9 & 41 \\
\hline 28 & 0,95 & 0,10 & 41 \\
\hline 39 & 0,10 & 0,10 & 42 \\
\hline 30 & 1,05 & 0,11 & 40 \\
\hline 31 & 1,10 & 0,11 & 42 \\
\hline 32 & 1,30 & 0,13 & 42 \\
\hline 33 & 1,65 & 0,17 & 47 \\
\hline 34 & 2,15 & 0,22 & 53 \\
\hline 35 & 3,2 & 0,32 & 57 \\
\hline 36 & 4,1 & 0,41 & 61 \\
\hline 37 & 5,25 & 0,53 & 65 \\
\hline 38 & 6,6 & 0,67 & 69 \\
\hline 39 & 8,55 & 0,90 & 69 \\
\hline 40 & 11,1 & 1,1 & 70 \\
\hline 41 & 14,4 & 1,4 & 70 \\
\hline 42 & 18,6 & 1,9 & 73 \\
\hline 43 & 24,1 & 2,4 & 74 \\
\hline 44 & 31,3 & 3,1 & 75 \\
\hline 45 & 40,5 & 4,1 & 75 \\
\hline 46 & 52,5 & 5,3 & 76 \\
\hline 47 & 68,0 & 6,8 & 76 \\
\hline 48 & 88,2 & 8,9 & 77 \\
\hline 49 & 114,3 & 11,4 & 77 \\
\hline \multicolumn{4}{|c|}{$\begin{array}{l}\text { Les anomalies chromosomiques représentées dans ce tableau sont la trisomie } 18 \text { (Syndrome d'Edwards), } \\
\text { trisomie } 13 \text { (Syndrome de Patau), le Syndrome de Klinefelter }(X X Y) \text {, le Syndrome du double } X \text { mâle }(X Y Y) \text {, } \\
\text { Syndrome de Turner }(X 0) \text {. }\end{array}$} \\
\hline
\end{tabular}

Source : Hook B. (1981). Rates of Chromosome abnormalities at Different Maternal Age. Obstetrics \& Gynecology, vol. 58, p. 282-285. 
ANNEXE 4

INSTRUMENT DE MESURE POUR LA COLLECTE DES CARYOTYPES

Annee/naissance Mois/naissance No. dossier

Lieu/naissance

No. caryotype

Configuration chromosomique :

Famille/propositus

Propositus

Âge

\begin{tabular}{|c|c|c|c|}
\hline Mère & Âge & L.N. & $\mathbf{M}$ \\
\hline Père & Âge & N & $\mathrm{M}$ \\
\hline
\end{tabular}

Données supplémentaires :

Commentaires :

Tégende : L.N. = Lieu naissance

$\mathbf{M}=\quad$ Marie 


\section{ANNEXE 5}

\section{Collecte des données effectuée par les médecins - Identification du proposant}

Méci $=s t$ lon depuls un nols

Typo se raslogreonle reçue depuls un nois

mpladles infectleuses antörleuras

MISTOIFE FAMLIAL:

hç du père

f-irss et sceurs

fils $=t$ thlles

Maladies congennltales sans la fanllie ou la porstré

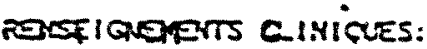

Dévoleppement staru- o-pondéral

Psrehl sate

contormation de la téte

Yeux

Bouche

Therax

Orgenes gönito-urinaires
Oreilles

Nez

Abdomen

Mrmbres supérlours

matres Iavariours

Systïn neuro-nusculalse

Srstim cardic-vas uulaite

Aetres parriculartiós

DIAGNOSTIC CHMIE PCYISOIES:

\section{GENETLUE HLARI:E COEMNOE AMATOMIE PATHOOSI QUE}

Source : Département de pathologie/Centre hospitalier Chicoutimi 


\section{MÉTHODES DE DIAGNOSTIC PRÉNATAL}

\section{ÉCHOGRA PHIE}

\section{Définition}

Projection d'ondes électromagnétiques sur l'abdomen dont la réflexion sur les structures osseuses produit une image qui apparâ̂t sur un écran cathodique.

\section{Fonctions}

Estimation de la DPA, identification de grossesse multiple, dépistage de malformation, localisation du placenta, évaluation de la quantité du liquide amniotique, évaluation de retard de croissance, profil biophysique du foetus.

Période de gestation

Vers la $18^{\mathrm{c}}$ semaine (examen de routine).

Risques pour la santé de la mère et de l'enfant

Aucun risque identifié jusqu'à maintenant.

\section{Conséquences psychosociales}

Favorise l'attachement mère/foetus, père/foetus, facilite l'adaptation à la grossesse, facilite l'intégration et la participation du père, favorise la fidélité aux recommandations thérapeutiques. 


\section{AMNIOCENTÈSE}

\section{Définition}

Ponction du liquide amniotique à l'aide d'une seringue introduite à travers la paroi abdominale. Se fait sous échographie.

\section{Fonctions}

Caryotype, maladies héréditaires ou métaboliques, maladies reliées aux chromosomes sexuels, étude de l'ADN par méthode d'amplification génétique, dosage d'enzyme ou de protéines.

\section{Période de gestation}

Entre 16 et 18 semaines. Délai d'attente des résultats : de 3 à 4 semaines.

\section{Risques pour la santé de la mère et de l'enfant}

Procédure relativement sûre. Complications possibles : mort foetale, contractions utérines, perte du liquide amniotique, saignement, infection, blessure foetale.

\section{Conséquences psychosociales}

La connaissance du sexe modifie les perceptions parentales; phénomène de la " grossesse à l'essai "; pics d'anxiété la veille de l'examen et pendant la période d'attente des résultats.

\section{BIOPSIE}

\section{Définition}

Prélèvement d'un échantillon de tissu provenant d'un fragment de l'oeuf fertilisé en insérant un instrument à biopsie à l'intérieur du col jusqu'à l'utérus. Se fait sous échographie. 


\section{Fonctions}

Caryotype, maladies métaboliques ou héréditaires, maladies reliées aux chromosomes sexuels, aberrations chromosomiques, étude de l'ADN.

\section{Période de gestation}

Vers la $10^{\mathrm{e}}$ semaine. Délai d'attente des résultats : de 7 à 10 jours.

\section{Risques pour la santé de la mère et de l'enfant}

Risques de perte foetale supérieurs de 1 à $2 \%$ à ceux de l'amniocentèse. Complications possibles : pertes sanguines, amniotique, avortement spontané.

\section{Conséquences psychosociales}

L'examen se fait au premier trimestre de la grossesse, période où la femme est encore ambivalente. L'investissement psychologique des parents au premier trimestre est moins important.

\section{FOETOSCOPIE}

\section{Définition}

Visualisation directe du foetus à travers un endoscope afin de prélever un échantillon de la peau, de l'urine ou du foie du foetus. Aspiration du sang foetal par le cordon ombilical. Se fait sous échographie.

\section{Fonctions}

Anémies héréditaires, hémophilie, maladie de Tay-Sachs, maladies cutanées graves, nanisme, état fonctionnel des reins, mesures thérapeutiques. 
Période de gestation

A partir de la $18^{\circ}$ semaine.

Risques pour la santé de la mère et de l'enfant

Risques très importants. Complications possibles : avortement spontané (de 3 à $5 \%$ ), prématurité.

\section{Conséquences psychosociales}

Cet examen est actuellement au stade de l'expérimentation. Les interventions thérapeutiques par rapport à la grossesse sur le foetus entraînent un questionnement éthique.

Source : Durand S., Goulet C. (1992). Le diagnostic prénatal. Mieux comprendre pour mieux agir. Nursing Québec, vol. 12, p. 15-24. 


\title{
ANNEXE 7
}

\section{GLOSSAIRE}

\begin{abstract}
Aberration chromosomique : les aberrations chromosomiques sont des anomalies du nombre ou de la structure des chromosomes (Solomon E. P., Davis P. W. 1981).
\end{abstract}

ADN satellite : ADN hautement répétitif , c'est-à-dire constitué d'une séquence de bases répétée en tandem un grand nombre de fois dans le génome.

Anaploïdie : l'anaploïdie définit un état où le nombre des chromosomes n'est pas exactement un multiple du nombre haploïde (23 chez l'humain) (Solomon E. P., Davis P. W. 1981)

Une anaploïdie peut consister en un chromosome surnuméraire de sorte que l'un des jeux d'homologues d'un individu est formé de 3 chromosomes (une trisomie) au lieu de 2 (Solomon E. P., Davis P. W. 1981).

Autosome : un autosome est un chromosome autre qu'un chromosome sexuel (X ou Y).

Caryotype : le caryotype est l'ensemble des chromosomes contenu dans le noyau de chacune des cellules d'un être vivant (Serre J-L., Feingold J., Gallano P., Boué J., Boué A. 1983). Le caryotype est la représentation (dessin ou microphotographie) de 
l'arrangement chromosomique, c'est-à-dire nombre, dimensions relatives et morphologie des chromosomes (Fattorusso V., Ritter O. 1986). Le caryotype est un assortiment chromosomique d'une cellule somatique (Solomon E. P., Davis P. W. 1981). Le caryotype humain normal comprend 46 chromosomes répartis en 22 paires d'autosomes et une paire de gonosomes ou chromosomes sexuels (XX pour le sexe femelle, $\mathrm{XY}$ pour le sexe mâle). Un caryotype normal présente des caractères propres à chaque espèce en nombre et structure de chromosomes. Un caryotype normal se veut diploïde (2n); il est constitué de la composition de deux matériels (stocks) haploïdes (n) contenus dans chacun des gamètes. Ces cellules sexuelles ne contiennent donc qu'un représentant de chacune des paires de chromosomes du parent donneur.

Caryotype haploïde : caryotype comprenant un seul exemplaire de chaque chromosome.

Centimorgan : un centimorgan est une unité d'évaluation de recombinaison qui mesure la distance pour laquelle on observe $1 \%$ de recombinaison. La taille de la carte génétique diffère selon le sexe (De Braekeleer M. 1989).

Chiasma : endroits où les chromatides restent en contact alors qu'elles sont séparées ailleurs et où les crossing-over ont lieu. 
Chromosome métacentrique : chromosome dont le bras court « $p$ » a la même taille que le bras long « q " (Mattei J. F., Mattei M. G. 1986).

Enjambement des chromosomes (cross-over) : entrecroisement des chromosomes avec échange des segments et recombinaison des gènes portés par ces segments. Il en résulte que des gènes d'un chromosome peuvent être échangés avec leurs allèles (Fattorusso V., Ritter O. 1986).

Gamète : un gamète est un spermatozoïde ou une ovule.

Génome : un génome est l'ensemble des gènes contenus dans la dotation chromosomique d'un gamète (haploïde) qui est chez l'homme de 23 chromosomes. Les cellules somatiques (diploïdes) qui contiennent 23 paires de chromosomes possèdent normalement deux génomes (Fattorusso V., Ritter O. 1986).

Génotype : un génotype est l'ensemble des gènes contenus dans les cellules d'un individu ou d'un groupe d'individus génétiquement identiques. La correspondance entre le génotype (ensemble des gènes) et le phénotype (ensemble des caractères apparents) dépendent de la pénétrance des gènes et de l'environnement (Fattorusso V., Ritter $O$. 1986). 
Gonosome : un gonosome est un chromosome sexuel $\mathrm{X}$ ou $\mathrm{Y}$.

Hybridation : réassociation de chaînes d'acides nucléiques complémentaires (Mattei J. F., Mattei M. G. 1986).

Isochrome : chromosome résultant d'une mauvaise division du centromère et constitué de deux bras identiques : deux fois le bras court, ou deux fois le bras long. Un isochrome est un chromosome anormal qui possède deux bras égaux et identiques génétiquement. Il se forme le plus souvent par division transversale du centromère lors de la mitose (alors que la division centromérique normale se fait dans le sens de la longueur) suivie d'une réduplication des segments télomériques lors de l'interphase suivante. Ce type de chromosome se retrouve dans les cellules cancéreuses.

Formule chromosomique : i (18q).

Marqueur génétique : le marqueur génétique est une variation génétique qui s'exprime soit dans la composition protéique ou dans le phénotype, et que l'on peut suivre de génération en génération (Patterson D. 1994).

Méiose : la méiose est une division cellulaire réductionnelle, celle qui permet de prołuire des gamètes possédant un nombre réduit de chromosomes, le nombre haploïde. (Solomon E.P., Davis P.W. 1981). La méiose est un processus de division cellulaire 
observé dans les testicules et les ovaires aboutissant à la réduction de moitié du nombre de chromosomes dans les gamètes. La première phase est dite « réductionnelle , parce que la cellule mère diploïde ( $2 n$ ) ayant 46 chromosomes, se divise en 2 cellules haploïdes (n) n'ayant que 23 chromosomes; pendant cette phase, il y a redistribution de gènes semblables d'origine matemelle et paternelle. La deuxième phase dite « équationnelle ", aboutit à la formation de 4 cellules filles haploïdes (n) qui deviennent 4 spermatozoïdes chez l'homme ou, chez la femme, un ovule (les 3 autres cellules haploïdes dégénèrent spontanément).

La méiose est un procédé de division cellulaire qui implique une réduction du nombre des chromosomes lors de la gamétogenèse (Serre J-L. et coll. 1983).

Monosomie : une monosomie est la présence d'un seul membre d'une paire de chromosomes, dont le nombre total est de 45 (Fattorusso V., Ritter O. 1986).

Formule chromosomique d'une monosomie 21: 45, XY,-21.

Mutation : changement brusque et permanent d'un ou de plusieurs caractères héréditaires survenant spontanément ou provoqué par divers agents (radiations, médicaments, virus). (Fattorusso V., Ritter O. 1986).

Changement des caractères d'un organisme dû à une altération transmissible de son matériel génétique (Solomon E. P., Davis P. W. 1981). 
Pénétrance : la pénétrance est la fréquence de la manifestation d'un gène dominant chez les hétérozygotes ou d'un gène récessif chez les homozygotes. La pénétrance dépend du génotype mais aussi de l'influence du milieu extérieur sur l'action des gènes (Fattorusso V., Ritter O. 1986).

Polyploïdie : la polyploïdie consiste en une juxtaposition de plus de deux stocks haploïdes de chromosomes; on peut observer ainsi des triploïdies ( $3 \mathrm{n}$ ), par exemple dans le cas d'une double fertilisation d'un gamète d'un des sexes par deux gamètes de l'autre sexe, ou des tétraploïdies.

Ségrégation : la ségrégation est une inégale fréquence des gamètes parentaux.

Sonde : marquage radio-actif d'une séquence d'ADN. Sonde purifiée : fragment d'ADN inséré dans un plasmide.

Tétrasomie : présence de quatre chromosomes au lieu de deux. 